\title{
Photoexcited transients in disordered semiconductors: Quantum coherence at very short to intermediate times
}

\author{
A. Kalvová \\ Institute of Physics, Academy of Sciences of the Czech Republic, Na Slovance 2, CZ-182 21 Praha \\ 8, Czech Republic \\ B. Velický \\ Institute of Physics, Academy of Sciences of the Czech Republic, Na Slovance 2, CZ-182 21 Praha \\ 8, Czech Republic \\ and \\ Faculty of Mathematics and Physics, Charles University, Ke Karlovu 5, CZ-121 16 Praha 2, \\ Czech Republic
}

\begin{abstract}
We study theoretically electron transients in semiconductor alloys excited by light pulses shorter than 100 femtoseconds and tuned above the absorption edge during and shortly after the pulse, when disorder scattering is dominant. We use non-equilibrium Green functions employing the field-dependent selfconsistent Born approximation. The propagators and the particle correlation function are obtained by a direct numerical solution of the Dyson equations in differential form. For the purely elastic scattering in our model system the solution procedures for the retarded propagator and for the correlation function can be decoupled.The propagator is used as an input in calculating the correlation function.

Numerical results combined with a cumulant expansion permit to separate in a consistent fashion the dark and the induced parts of the self-energy. The dark behavior reduces to propagation of strongly damped quasi-particles; the field induced self-energy leads to an additional time non-local coherence.

The particle correlation function is formed by a coherent transient and an incoherent back-scattered component. The particle number is conserved only if the field induced coherence is fully incorporated. The transient polarization and the energy balance are also obtained and interpreted.
\end{abstract}

78.47.+p, 71.23.-k, 72.10.Bg

Typeset using REVTEX 


\section{INTRODUCTION}

The response of electrons in a semiconductor to a strong short optical pulse is an important case of transient non-equilibrium processes in many body systems. It is discussed in detail in monographs by Haug and Jauho [1] and by Bonitz [2]. Step by step, the technique of non-equilibrium Green's functions (NGF) has been recognized as the most reliable and physically most transparent method for handling strongly non-equilibrium processes. However, because of the prohibitive requirements on computational means, its practical use was until recently connected with various approximation schemes reducing the full Kadanoff-Baym equations (KBE)or their equivalents to simplified quantum transport equations by means of various decouplings ("ansatzes"). The generic ansatz appears already in the classical works by Kadanoff and Baym [3] and by Keldysh [4], and it was followed by a number of its refinements. This work was very successful in describing a wide variety of experimental data almost quantitatively. Still, uncertainties concerning the validity and more subtle properties of the additional approximations remain.

Since early times, however, there existed an important line of research [5], [6] on the direct solution of the KBE for transients, both in electron systems and in nuclear matter, where it was mandatory to use the NGF and related quantities as two time functions. More recently, efforts have concentrated precisely on a direct solution of the full NGF incorporating both the electron-phonon and electron-electron interactions, as discussed already in Ref. [2] and expounded in more detail in the proceedings [7]. The need to work with two time functions limits the physical approximations for the self-energies, as analyzed in the general references given above.

We will consider the third principal mechanism of electron scattering, caused by a static random potential. In random semiconductor alloys the strongest scattering is often caused just by the disorder, which then dominates the early stages of the transient response to a pulsed disturbance. The case of disorder has some interesting special properties while providing an excellent model situation to study general aspects of non-linear transients [8] [9] [10] [11]. This is well illustrated on our two-band model semiconductor with disorder only acting in the conduction band [12]. A dipole optical transition transfers a valence electron with a sharp wave vector value $k$ to the conduction band. For one alloy configuration, the conduction states are random and have no wave vector at all. In the one-electron picture, this is a fully unitary evolution in which the transition is made to many of the random states with a full preservation of the quantum coherence. Alternatively, a configuration average is performed [13] accounting for the fact that the macroscopic response of the electrons is self-averaging [14]. The static random fluctuations of the alloy potential play the role of the quantum field fluctuations of the many body theory ("frozen phonons"). Now, the transitions preserve the $k$-vector, but the final states are renormalized and decaying, independently of the excitation process or of the electron distribution. In analogy with the phonon case, this may be termed a dark polaron effect. The optical jump injects an electron into a bare Bloch conduction state, and its transition into the dressed state (formation process) plays an important role for the short time dynamics of the coherent excitation. At longer times, the incoherent back-scattering sets on to compensate for the decay of the coherent population. All these features coincide with those occurring in a genuine quantum field case, and it appears that for the disorder case the full NGF treatment is the only reliable approach. In 
paper [8], we have developed the basic theory for NGF in the presence of external fields and alloy scattering and solved the equations of motion in the coherent potential approximation (CPA, for the equilibrium case and linear response see Ref. [13]) in a closed form. The solution was limited to an unrealistic case of a sharp onset of steady illumination, however. In [9], this solution was used for a numerical test of one of the successful ansatzes, the so-called GKBA [15]. In [10], an adiabatic approximation was developed for slowly varying pulses, employing the mixed time-energy representation, and in [11], we were able to present early results for retarded propagators in the presence of pulses of a general shape.

This paper extends our previous work to a full computation of the response to an arbitrary pulse, although only in the weak scattering limit of the CPA, the self-consistent Born approximation (SCBA).

The alloy scattering on a static potential is specific in that it is strictly elastic and instantaneous, with no internal time structure. This has an important consequence. The electron propagators do not depend on the electron distribution, being basically a configuration averaged one-electron evolution operator. The propagators can thus be studied, analytically and numerically as well, in an independent and comparatively easy step. In the second step yielding the full NGF, the propagators serve as an input and it is sufficient to solve a single linear equation for, say, the particle correlation function, instead of the usual set of two coupled non-linear Kadanoff-Baym equations.

On top of that, the use of this asymmetric set of Dyson equations [16 permits a direct interpretation of some features of the non-equilibrium process. By concentrating first on the propagators, we can study in detail the formation stage and the transition to the long time ("quasi-particle") regime. The self-energy can be separated into its dark part and the induced component, which reflects the coherent coupling between the excitation and the scattering. Proper inclusion of this coherence effect appears to be essential to keep the theory conserving and consistent. This becomes apparent in the behavior of the particle correlation function, in the loss of coherence in the non-equilibrium electron distribution and in the validity of conservation laws.

We start, in Sec. [I, by describing chemical disorder in semiconductor alloys (IIA) which serves to justify the simple two band model with a short-range disorder of atomic levels acting only in the conduction band, as introduced in एᄑ. The effect of the alloy disorder in the dark is discussed in Sec. IIII. First, the SCBA is introduced and the renormalized bands are obtained (IIIA). For an actual choice of the model parameters, the renormalized bands, their damping, and the weak field one-photon resonance conditions are presented ([IIB). The next subsection $\amalg I \mathrm{C}$ deals with the dark propagators in the time domain. The formation process leading from a bare electron to a quasi-particle is analyzed. The SCBA is compared with the exact short time cumulant expansion of the GF and the formation time is estimated.

In Sec. IV, the action of the light pulse is included. Specific properties of NGF in disordered systems are introduced in IVA. General relations for the Langreth-Wilkins form of NGF are in IVA 1. Next two subsections एVA2, एVA3 introduce the GF for a single alloy configuration and its configuration average. A closed form of the perturbation expansion is obtained. In IVB, the explicit equations of motion for all components of the NGF are given (IVB 1). In IVB 2 we separate the dark polaron effect from the light induced self-energy. Its properties are analyzed in IVB3, where the short time expansion is extended also to the 
excited system. The meaning of the dark quasi-particle approximation is clarified in IV B 4. The actual computational schemes for both Dyson equations are presented in Subsec. IVC.

Sec. $\nabla$ is devoted to numerical results. The actual choice of the pulse is specified (Subsec. $\mathrm{VA})$. The retarded propagator is presented in $\mathrm{VB}$. An example of the induced self-energy is shown in Subsec. VB1 and its role for the propagator is demonstrated in VB2. The related coherence and time non-locality leads to a breakdown of the semi-group property of the propagator, Subsec. VB3. The computed behavior of the particle correlation function is described in $\nabla \mathrm{V}$, presenting $\Sigma^{<}\left(t, t^{\prime}\right)$ in $\overline{V C} 1$ and an example of $G^{<}\left(\mathrm{k} ; t, t^{\prime}\right)$ itself in $\overline{V C 2}$.

Physically, we are particularly interested in the averaged one-particle density matrix, and in the observable averages computed with its aid. Sec. $\nabla \mathbb{1}$ is divided into two subsections. In the first one, VIA, we consider the connection between the one electron density matrix and the particle correlation function, construction of the precursor kinetic equation and a proof of the particle number conservation in SCBA (VIA 1). Several observables and their average values are treated in VIA2. In the last subsection VIB we show the results of the corresponding computations. First, individual matrix elements of $\varrho(t)$ are shown and discussed in VIB 1. The last part VIB2 concerns observables. The light-disorder coherent coupling conditions the particle number conservation which hinges upon a full inclusion of the induced self-energy in the computation. The energy is not dissipated in the model, only transferred between the pulse and the electrons, while the dephasing of the interband offdiagonal components of the polarization need not be introduced by hand, as it appears to be linked with pronounced cancellations of the oscillatory integrand in the trace yielding the total polarization.

Two appendices outline the steps necessary to incorporate into the theory random initial conditions (A) and observables represented by random operators (B).

\section{MODEL DESCRIPTION OF THE SYSTEM}

In this paper, we use the model semiconductor alloy introduced previously [8], [10]. The simplifications inherent to the model bring it to a close parallel to two-band models with electron-phonon interaction [1]. Static alloy disorder in this picture may be understood in terms of "frozen phonons". The model belongs to the family of "independent band models" used to study optical transport in disordered systems [12].

\section{A. Electrons in semiconductor alloys}

One aim of this paper is to turn attention to the importance of disorder effects in the optical response to short pulses; once the excitation will take place at regions of the band structure even moderately beyond the absorption edge, the alloy scattering in a mixed crystal will frequently become stronger than either the $e-e$ or the $e-p$ collisions and it will represent the principal mechanism of relaxation of the photoexcited population during the initial period. As an example, consider the zinc blende semiconductors. Even for a conservative choice, the $\mathrm{Ga}_{1-c} \mathrm{Al}_{c}$ As system on the GaAs side, $c \leq 0.1$, pronounced alloy scattering effects are predicted [17]. Stronger effects still can be expected for other systems, in particular some of the II-VI alloys [18]. In all such materials, the basic alloying mechanism 
is an isoelectronic substitution, by atoms from the same group of the periodic system. As a consequence, the random one-electron potential in the alloy is dominated by short range, basically site-localized fluctuations. In an orbital picture, this is best captured by a oneelectron Hamiltonian in a localized orbital ("tight binding") representation [13], which is by now standard, and considered quantitative [19], [20]. The tight binding Hamiltonian in the minimum basis of quasiatomic orbitals will typically involve (i) non-random off-diagonal hopping matrix elements responsible for bonding between the $s p^{3}$ hybrids, and for creation of the corresponding hybridization gap between the valence and the conduction bands, and (ii) random diagonal elements, quasiatomic levels $\epsilon_{\ell}$, with as many admissible values, as are the alloy components (see, for example, [18]).In the case of $\mathrm{Ga}_{1-c} \mathrm{Al}_{c} \mathrm{As}$, the doping proceeds only on the cationic sublattice. In the Harrison parametrization [19]:

$$
\begin{gathered}
\epsilon_{4 s}^{\mathrm{Ga}}=-11.37 \mathrm{eV}, \epsilon_{3 s}^{\mathrm{Al}}=-10.11 \mathrm{eV} \\
\epsilon_{4 p}^{\mathrm{Ga}}=-4.90 \mathrm{eV}, \epsilon_{3 p}^{\mathrm{Al}}=-4.86 \mathrm{eV} .
\end{gathered}
$$

If the alloy is random, that is, without any short or long range order, the random potential is fully characterized by the $\epsilon$ values and the concentration $c$. In particular, the ratios (LEVEL DIFFERENCE)/BANDWIDTH determine the alloy regime. If they are much less than one (as is the case for $\mathrm{Ga}_{1-c} \mathrm{Al}_{c} \mathrm{As}$ ), a perturbation approach is justified. The $\ell$-contribution to the level broadening is then proportional to the projected density of states of a given symmetry. In general, spectral weight of each orbital is divided between the conduction bands and the valence bands, so that, say, the disorder of the cation $s$ levels affects both. The projected spectral weights are distributed unevenly, however. Around the gap, where

the optical excitation is assumed to act, the conduction band is predominantly composed of cation states, and these mostly with the $s$-symmetry. The top of the valence band, on the contrary, is nearly anion $p$ by nature. The cationic disorder, as a result, acts much more strongly at the bottom of the conduction band than at the top of the valence band.

\section{B. Two band model}

On the basis of these remarks, we will now develop a simplified model of the electronic structure of this system as used in Refs. [8], [10].

1. We consider a two-band semiconductor with the gap between two isotropic parabolic band edges at the center of the Brillouin zone ("standard band structure")

2. All many-body interactions are ignored

In general, there are three mechanisms by which the disorder couples both bands: the $c-v$ chemical hybridization mixing the conduction and the valence states, a statistical correlation between the electrons moving in both bands caused by the atomic disorder configuration they have in common, and a dynamical transfer of disorder between the bands due to the mixing of optically coupled valence and conduction states. We will eliminate both static coupling mechanisms by hypothesis.

3. The disorder acts in each band separately, not mixing states of both bands ("independent bands") 
4. The disorder in the valence band is neglected ("cationic doping").

5. The effect of the optical pulse is restricted to a non-random interband dipole coupling treated in the rotating wave approximation (RWA).

This model appears as reasonable for an optical excitation slightly beyond the absorption edge, but the band structure has to be extended to the whole Brillouin zone for two reasons: $\diamond$ the single site nature of the disorder spreads its effect homogeneously over the whole Brillouin zone, $\diamond$ a short strong optical pulse acts in an extended part of the Brillouin zone, (E-t uncertainty). This becomes apparent, for example, in the band renormalization caused by the disorder, and, in particular, in the short time quasi-particle formation process. The band parts remote from the Brillouin zone center can be described rather schematically, however, as only their gross properties enter the problem.

Disorder does not mix the two bands. The band projectors of these "independent bands" are non-random and diagonal in both the Bloch and the Wannier (site) basis:

$$
P_{b}=\sum_{\mathrm{BZ}}|b \mathrm{k}\rangle\left\langle b \mathrm{k}\left|=\sum_{\text {lattice }}\right| b i\right\rangle\langle b i|, \quad b=c, v .
$$

The full one-electron Hamiltonian for one configuration of an $\mathrm{A}_{1-c} \mathrm{~B}_{c}$ alloy has the following structure:

$$
\mathcal{H}=W_{v}+W_{c}+\mathcal{V}_{c}+U(t)
$$

The non-random configuration independent or averaged quantities are denoted by italics, while the configuration dependent ones by rounded (" \cal ") characters.

Here, $W_{v}+W_{c}$ is the Bloch Hamiltonian of the A crystal. Both band Hamiltonians are diagonal in the Bloch basis:

$$
W_{b}=P_{b} W_{b} P_{b}=\sum_{k}|b k\rangle \epsilon_{b}(k)\langle b k|, \quad b=c, v
$$

The random potential acts only in the conduction band and it is site diagonal:

$$
\mathcal{V}_{c}=P_{c} \mathcal{V}_{c} P_{c}=\sum_{i}|c i\rangle \epsilon_{i}\langle c i|
$$

Here, $\epsilon_{i}$ are quasiatomic level shifts $\epsilon^{A}$ or $\epsilon^{B}$.

Finally, $U(t)$ is a non-random inter-band dipole coupling of the pulse to the electrons, whose electric field is a linearly polarized harmonic wave with a basic frequency $\Omega$ and an envelope $\mathbf{E}_{\mathrm{M}} \Phi(t)$ with $\Phi \geq 0, \max \Phi=1, \mathbf{E}_{\mathrm{M}}$ is the peak value of the field:

$$
\mathbf{E}(t)=\mathbf{E}_{\mathrm{M}} \cos (\Omega t) \Phi(t) \mathbf{e}
$$

Assuming validity of the Rotating wave approximation (RWA), and making at the same time the approximation of a $k$-independent transition matrix element, we get for $U(t)$ :

$$
\begin{aligned}
& U(t)= U_{c v}(t)+U_{v c}(t)=-e\left(\mathbf{r}_{c v}+\mathbf{r}_{v c}\right) \mathbf{E}(t) \\
& \stackrel{\mathrm{RWA}}{\longrightarrow}-\Phi(t)\left\{\sum_{k}|c \mathrm{k}\rangle Q \mathrm{e}^{-\mathrm{i} \Omega t}\left\langle v \mathrm{k}\left|+\sum_{k}\right| v \mathrm{k}\right\rangle Q^{*} \mathrm{e}^{\mathrm{i} \Omega t}\langle c \mathrm{k}|\right\} \\
& \quad \mathrm{r}_{c v}=P_{c} \mathrm{r} P_{v}, \text { etc., } \quad Q=\frac{1}{2} e \mathrm{E}_{\mathrm{M}}\langle c \mathrm{k} \simeq 0|(\mathbf{e}, \mathbf{r})| v \mathrm{k} \simeq 0\rangle
\end{aligned}
$$


The coupling strength parameter $Q$ can be made real positive, $Q=Q^{*}>0$ by rephasing. RWA allows to make a time-dependent unitary transformation

$$
O=P_{c}+P_{v} \mathrm{e}^{-\mathrm{i} \Omega t}
$$

of the Hamiltonian (the Galitskii transformation [21]) to eliminate the rapid $\Omega$ oscillations of $U(t)$. Thus, $\mathcal{H}(t) \stackrel{O}{\longrightarrow} \tilde{\mathcal{H}}=O \mathcal{H} O^{\dagger}-O \mathrm{i} \hbar \partial_{t} O^{\dagger}$ :

$$
\begin{aligned}
\tilde{\mathcal{H}}(t)= & \tilde{W}_{v}+W_{c}+\mathcal{V}_{c}+\tilde{U}(t) \\
& \tilde{\epsilon}_{v}(\mathrm{k})=\epsilon_{v}(\mathrm{k})+\hbar \Omega \\
\tilde{U}(t)= & -Q \Phi(t)\left\{\sum_{k}|c \mathrm{k}\rangle\left\langle v \mathrm{k}\left|+\sum_{k}\right| v \mathrm{k}\right\rangle\langle c \mathrm{k}|\right\}
\end{aligned}
$$

The valence band is displaced by $\hbar \Omega$ upwards on the transformation. The interaction with light appears now as a transient hybridization of the transformed bands giving rise to the band splitting sometimes called the "Galitskii gap".

From now on, we will work in the Galitskii picture dropping the tilde accents over the operators, but we will keep the tildes to indicate the shifted valence band energies.

Denoting the usual configuration average by $\langle\ldots\rangle$, we may introduce the mean-field Hamiltonian $H_{\mathrm{MF}}$ and the configuration dependent random field $\mathcal{D}$ :

$$
\begin{aligned}
\mathcal{H}(t) & =H_{\mathrm{MF}}(t)+\mathcal{D}(t) \\
H_{\mathrm{MF}}(t) & =\langle\mathcal{H}(t)\rangle \\
\langle\mathcal{D}(t)\rangle & =0
\end{aligned}
$$

In our model, $U(t)$ is non-random, while $\mathcal{V}$ is time independent. Forming $H_{\mathrm{MF}}$ then means that the virtual crystal part of $\mathcal{V}_{c}$ responsible for the rigid band shift is transferred to the renormalized conduction band $\bar{W}_{c}$ :

$$
H_{\mathrm{MF}}=W_{v}+\bar{W}_{c}+U(t)
$$

with

$$
\begin{aligned}
\bar{W}_{c} & =\sum_{k}|c k\rangle \overbrace{\epsilon_{c}(\mathrm{k})+\langle\epsilon\rangle}^{\epsilon_{\mathrm{MF}}(\mathrm{k})}\langle c k| \\
\mathcal{D} & =\sum_{i}|c i\rangle \underbrace{\left(\epsilon_{i}-\langle\epsilon\rangle\right)}_{d_{i}}\langle c i|
\end{aligned}
$$

where $\langle\epsilon\rangle$ is the average atomic energy $(1-c) \epsilon^{A}+c \epsilon^{B}$. The A crystal will be doped with $\mathrm{B}$ atoms, thus we choose

$$
\epsilon^{A}=0, \quad \epsilon^{B}=\delta, \quad\langle\epsilon\rangle=c \cdot \delta
$$

There are thus two alloy disorder parameters, concentration $c$ and the level fluctuation $\delta$. 


\section{DARK ALLOY}

The disorder scattering is present even without the optical excitation, and the $c$-band states are correspondingly distorted. The excitation transfers electrons into these disorder dressed states, and the behavior of the light induced transition states can best be described differentially with respect to the dark dressed states. This situation is similar to the dark polaron effect in the initial state [22]. The poles of the renormalized dark alloy bands define the weak field resonance transitions, but for strong pulses, the short time deviations from the pole (quasi-particle) behavior are essential.

In this section, we will consider this dark alloy case. This is an equilibrium situation characterized by a time independent $\mathcal{H}$. While in general the electron response is conveniently expressed in terms of the non-equilibrium Green function, in equilibrium it is sufficient to consider the retarded propagator $G^{R}$ of one electron moving in the dark conduction band.

The propagator then depends just on the time difference $t-t^{\prime}$, and may be analyzed without invoking the NGF formalism in the spectral representation introduced by Fourier transformation:

$$
G^{R}(\mathrm{k}, t)=\int \frac{\mathrm{d} E}{2 \pi \hbar} G(\mathrm{k}, E+\mathrm{i} 0) \cdot \mathrm{e}^{-\mathrm{i} E t / \hbar}
$$

\section{A. Dark alloy bands in SCBA}

A single conduction band with diagonal disorder is easy for treatment in any single site approximation, as described in detail in [13. Here, we only sketch the Self-consistent Born approximation results. The Green function in the energy representation coincides with the configuration averaged resolvent, and is given by

$$
G(\mathrm{k}, z)=\frac{1}{z-\epsilon_{k}-\langle\epsilon\rangle-\Sigma(z)}
$$

Here, $z$ denotes the complex energy, $\epsilon_{k} \equiv \epsilon_{c}(\mathrm{k})$ and the SCBA self-energy does not depend on $\mathrm{k}$. It is determined from

$$
\begin{aligned}
\Sigma(z) & =\gamma \cdot F(z) \\
F(z) & =N^{-1} \sum_{k} G(k, z) \\
\gamma & =c(1-c) \delta^{2}
\end{aligned}
$$

The Nordheim parameter $\gamma$ measures the scattering strength of the random potential, $F$ is the local Green function(LGF); its imaginary part determines the conduction band DOS per cell by $g_{c}(E)=\mp \pi^{-1} \operatorname{Im} F(E \pm \mathrm{i} 0)$. Combining Eqs. (16) and (17), we express this local GF in the alloy in terms of $F_{o}$, the pure A crystal LGF, with a shifted argument:

$$
F(z)=F_{o}(z-\langle\epsilon\rangle-\Sigma(z))
$$

The SCBA equation (17) reads explictly: 


$$
\Sigma(z)=\gamma \cdot F_{o}(z-\langle\epsilon\rangle-\Sigma(z))
$$

This can be solved by iteration. The dispersion law and the pole approximation, however, may be obtained explicitly. Let $\delta$ grow from zero to its actual value. The band energies turn adiabatically into the complex resonance energies, $\epsilon_{k} \equiv \epsilon_{c}(\mathrm{k}) \mapsto z_{k} \equiv z_{c}(\mathrm{k})$, given by the poles of the Green function (16) (on the analytic continuation to the non-physical sheet of the $z$-Riemann surface]:

$$
\begin{aligned}
G^{-1}\left(\mathrm{k}, z_{k}\right) & =0 \\
z_{k} & =\epsilon_{k}+\langle\epsilon\rangle+\Sigma\left(z_{k}\right)
\end{aligned}
$$

This holds for any $k$-independent self-energy. In the SCBA, (20) becomes an explicit expression for the SCBA dispersion law,

$$
z_{k}=\epsilon_{k}+\langle\epsilon\rangle+\gamma \cdot F_{o}\left(\epsilon_{k}+\mathrm{i} 0\right)
$$

The result looks like a most naive use of the non-selfconsistent Born approximation.

Next, we rewrite Eq. (16) into a residuum form,

$$
G(k, z)=\frac{Z_{k}}{\left(z-z_{k}\right)}+R(k, z)
$$

where $Z_{k}$ is the renormalization constant and $R(k, z)$ is regular in the neighborhood of the pole. From (19) and (20), we get also the renormalization constant explicitly:

$$
Z_{k} \equiv \frac{1}{1-\left.\frac{\mathrm{d}}{\mathrm{d} z} \Sigma(z)\right|_{z_{k}}}=1+\left.\gamma \cdot \frac{\mathrm{d}}{\mathrm{d} z} F_{o}(z)\right|_{\epsilon_{k}}
$$

\section{B. Parametrization of the dark alloy model}

A sufficient input for the dark alloy includes $\diamond$ alloy parameters, $\delta$ and $c$, $\diamond$ the $c$-band density of states per cell, $g_{o}$, from which the local GF $F_{o}$ can be obtained by the usual spectral representation

$$
F_{o}(z)=\int \mathrm{d} \eta \frac{1}{z-\eta} g_{o}(\eta)
$$

Because we will be interested mostly in the spectral region of the lower band edge, a model $g_{o}$ may be considered satisfactory, if it has the correct energy position of this edge and the curvature yielding the required (density) effective mass. Otherwise, it should have a proper bandwidth and it must satisfy the sum rule $\int \mathrm{d} \eta g_{o}(\eta)=1$. One easy way, adopted presently, to adjust the DOS to these requirements is to write it as an expansion in terms of the Tschebyshev polynomials of the second kind. An example of $F_{o}$ resulting from such procedure is shown in Fig.1. Details are given in an appendix to [10]. The parameters used were the band edge $\epsilon_{c}(\mathrm{k}=\Gamma) \equiv E_{G}^{A}=1.5 \mathrm{eV}$, the effective mass $m_{c}^{\times}=0.4 m_{e}$, the half-bandwidth $w_{c}=6 \mathrm{eV}$. 
The renormalized dispersion law $z_{k}=z_{c}(\mathrm{k})$ as a function of $\epsilon_{k}$, is given by (21). The renormalization has two parts: a rigid shift $\langle\epsilon\rangle=c \delta$ of the whole band proportional to the alloy concentration, in agreement with the known notion of the rigid band model [13], and a complex component $\gamma \cdot F_{o}\left(\epsilon_{k}+\mathrm{i} 0\right)$, whose real part gives the negative "polaron shift", and whose imaginary part is related with the electron life time by $\tau \approx \hbar / 2\left|\operatorname{Im} z_{k}\right|$. The polaron shift varies with concentration in the "Nordheim" fashion, proportionally to $c(1-c)$. It is not entirely rigid, which leads to an energy and concentration dependent renormalization of the effective mass. This effect is minor in our case, however.

In Fig. \#, we display by thick lines the renormalized quasi-particle energies $z_{k}$ as a function of the bare band energy $\epsilon_{k}$, taking $\delta=0.84 \mathrm{eV}$, and varying $c$ in the range $0 \div$ 0.5. The energy dependent band broadening is depicted in Fig. 2 by lining each of the renormalized dispersion laws $\operatorname{Re} z_{k}$ by thin lines at a distance $\pm \operatorname{Im} z_{k}$. The rigid band energies are shown by dashed lines, and the polaron shift is given by the vertical distance between the full and the dashed lines.

The corresponding renormalization constant is obtained from (23) and is shown in Fig. 3. This quantity is complex, and its module appears to be greater than 1, while the phase shift is negative. Thus, the long time behavior of the quasi-particle state will have the appearance of the simple Weisskopf-Wigner decaying state lagging somewhat behind in time.

Returning to Fig. 目, we discuss the plots (-.-.) of the shifted valence band. With the basic frequency of the pulse specified,the valence band is shifted to $\tilde{\epsilon}_{v}(\mathrm{k})=\epsilon_{v}(\mathrm{k})+\hbar \Omega$. Its crossing with the renormalized conduction band is the point of the weak field one-photon resonance (vertical transition):

$$
z_{k}^{\times} \equiv z_{c}\left(\mathrm{k}^{\times}\right)=\epsilon_{v}\left(\mathrm{k}^{\times}\right)+\hbar \Omega
$$

In the parabolic region, the two bare bands are related by

$$
\epsilon_{v}(\mathrm{k})=\frac{m_{c}}{m_{v}}\left(E_{G}^{A}-\epsilon_{c}(\mathrm{k})\right)
$$

(For simplicity, we will extend this as a definition for the entire model valence band.) Choosing $m_{v}=0.6 m_{e}$ and taking first $\hbar \Omega=2 \mathrm{eV}$, we plot in Fig. 2 the corresponding line. The crossing points with the renormalized $c$-bands define the electron and the hole energy at resonance, and also the respective excess energies easy to read as the separations between the crossing point and the band edges at the vertical axis. The bare energy of the crossing point serves to specify the position $\mathrm{k}^{\times}$of the resonance in the Brillouin zone.

The crossing points and the excess energies vary with the alloy concentration for a fixed $\Omega$, and it would be preferable to adjust the basic frequency used, so that either the crossing point $\mathrm{k}^{\times}$or the conduction band excess energy be kept fixed. The vertical line laid through the $c=0$ crossing point defines, how the frequency should be varied with concentration in order to excite resonantly always the same part of the Brillouin zone. In practice, a nearly equivalent way of tuning the frequency $\Omega \rightarrow \Omega^{\times}(c)$ may be to keep the excess energy equal to the difference $\hbar \Omega^{\times}(c)-E_{G}(c)$ constant, at least in the virtual crystal sense. The numbers corresponding to Fig. 2 are listed in Tab. [ and give an estimate of the importance of the alloy effects: It is seen that even the rather moderate alloy parameters produce a strong effect, in particular for the electron lifetimes. For stronger alloy scattering, the quasi-particle concept becomes useless, because it would be damped before completion of its formation. 


\section{Time dependence of the dark Green function}

Inserting the pole representation (22)into the Eq. (15), we have

$$
G^{R}(\mathrm{k}, t)=\frac{1}{\mathrm{i} \hbar} Z_{k} \mathrm{e}^{-\frac{\mathrm{i}}{\hbar} z_{k} t}+\int \frac{\mathrm{d} E}{2 \pi \hbar} R(\mathrm{k}, E+\mathrm{i} 0) \cdot \mathrm{e}^{-\mathrm{i} E t / \hbar}
$$

The first term prevails under typical conditions at sufficiently long times, and this corresponds to the quasi-particle behavior.

To study arbitrary times, it is convenient to use an 'interaction picture' for $G^{R}$ by factorizing off the mean field (virtual crystal) propagator. For every $\mathrm{k}$, we define ( $c f$. ([13))

$$
\begin{aligned}
\epsilon_{\mathrm{MF}} & =\epsilon_{k}+\langle\epsilon\rangle \\
G^{R}(\mathrm{k}, t) & \equiv \frac{1}{\mathrm{i} \hbar} \mathrm{e}^{-\frac{\mathrm{i}}{\hbar} \epsilon_{\mathrm{MF}} t} \times \mathrm{e}^{-\frac{\mathrm{i}}{\hbar} \varsigma(t)}
\end{aligned}
$$

The additional factor $\mathrm{e}^{-\frac{\mathrm{i}}{\hbar} \varsigma(t)}$ describes the transformation to a quasi-particle state and its further evolution. We express it as a complex phase factor by introducing an "action" $\varsigma=$ Re $\varsigma+\mathrm{i} I m \varsigma$. Its real part describes the additional phase, the imaginary part measures the decay of the amplitude $\left|G^{R}\right|$. This identification is verified in the long time limit. By (27), the leading terms in $\varsigma$ represent the "polaron shift" $z_{k}^{\prime}-\epsilon_{\mathrm{MF}}$ and the damping $\left|z^{\prime \prime}\right| / \hbar$,

$$
\varsigma(t) \approx\left(z_{k}^{\prime}-\epsilon_{\mathrm{MF}}\right) t+\mathrm{i} z_{k}^{\prime \prime} t+\mathcal{O}(t)
$$

For short times, we may employ the cumulant expansion of $G$, described in [23], p.3, [24], Ch.2. that is to expand in powers of time:

$$
\begin{aligned}
\mathrm{e}^{-\frac{\mathrm{i}}{\hbar} \varsigma(t)} & =\sum_{p=0}^{\infty} \frac{(-\mathrm{i})^{p} t^{p}}{p ! \hbar^{p}} M_{p} \\
\varsigma(t) & =\mathrm{i} \hbar \sum_{p=1}^{\infty} \frac{(-\mathrm{i})^{p} t^{p}}{p ! \hbar^{p}} C_{p}
\end{aligned}
$$

Here, $M_{p}$ are moments of the spectral density of the state $|\mathbf{k}\rangle$ calculated with respect to $\epsilon_{\mathrm{MF}}$, while $C_{p}$ are the corresponding cumulants. The moments, at least for small $p$, can be calculated directly without difficulty, proceeding, for example, similarly as in [25]. There are two options. Either the moments are calculated exactly, employing the fact that $G^{R}$ coincides with the configuration averaged evolution operator up to a $1 / \mathrm{i} \hbar$ factor:

$$
\begin{aligned}
G^{R}(\mathrm{k}, t) & =\frac{1}{\mathrm{i} \hbar}\left\langle\mathrm{k}\left|\left\langle\mathrm{e}^{-\frac{\mathrm{i}}{\hbar} \mathcal{H} t}\right\rangle\right| \mathrm{k}\right\rangle \\
& =\frac{1}{\mathrm{i} \hbar} \mathrm{e}^{-\frac{\mathrm{i}}{\hbar} \epsilon_{\mathrm{MF}} t} \times\left\langle\mathrm{k}\left|\left\langle\mathrm{e}^{-\frac{\mathrm{i}}{\hbar}\left(\mathcal{H}-\epsilon_{\mathrm{MF}} 1_{\mathrm{qP}}\right) t}\right\rangle\right| \mathrm{k}\right\rangle \\
& =\frac{1}{\mathrm{i} \hbar} \mathrm{e}^{-\frac{\mathrm{i}}{\hbar} \epsilon_{\mathrm{MF}} t} \times \sum_{p=0}^{\infty} \frac{(-\mathrm{i})^{p} t^{p}}{p ! \hbar^{p}} \underbrace{\left\langle\mathrm{k}\left|\left\langle\left(\mathcal{H}-\epsilon_{\mathrm{MF}} 1_{\mathrm{op}}\right)^{p}\right\rangle\right| \mathrm{k}\right\rangle}_{M_{p}}
\end{aligned}
$$

Or the moments are determined within the SCBA. In that case, they should be identified with the coefficients of the Laurent expansion of $G(z)$ at infinity, 


$$
G(\mathrm{k}, z)=\sum_{p=0}^{\infty} \frac{M_{p}}{\left(z-\epsilon_{\mathrm{MF}}\right)^{p}}
$$

Now, we have to combine Eqs. (16), (19) and (24), using Laurent expansions of all quantities involved and determining the coefficients successively.

Once the moments are determined in either way, the cumulants are obtained by comparing both series in (32). The results for $p=0 \div 4$ are in the table [1; everything is expressed in terms of the moments of the $\mathrm{VC}$ density of states defined by $\mu_{p}^{\mathrm{VC}}(E)=$ $\int \mathrm{d} \bar{E} g_{o}(\bar{E}-\langle\epsilon\rangle)(\bar{E}-E)^{p}$ and of the alloy characteristics $c_{a}-c_{B}, \delta, \gamma$. It should be noted that because of the choice $E=\epsilon_{\mathrm{MF}}$ as the reference energy, the first moment and cumulant both vanish, $M_{1}=C_{1}=0$. The vanishing first moment simplifies the cumulant expressions in all orders: Up to $p=2$, the SCBA results and the exact ones are the same. Differences occur for higher $p$. The SCBA expressions use $\gamma$ as the only characteristic of disorder beyond the mean field, as implied by (17). In both approaches, the moments coincide with the cumulants up to the third order, and the first deviations occur in the fourth order as marked in boldface.

We plot an example of the time evolution of the "phase" in Fig. (1. The gradual onset of $\varsigma$ for the shortest times justifies the conjecture that the one electron excitation starts as a bare (mean field) particle and develops the cloud of "frozen phonons" only in the course of time:

$$
G^{R}(\mathrm{k}, t)=\frac{1}{\mathrm{i} \hbar} \mathrm{e}^{-\frac{\mathrm{i}}{\hbar} t\left(\epsilon_{\mathrm{MF}}-\frac{1}{6 \hbar^{2}} C_{3} \cdot t^{2}+\ldots\right)} \times \mathrm{e}^{-\frac{1}{2 \hbar^{2}} \gamma \cdot t^{2}+\ldots}
$$

This behavior prevails only for very short times, and it is succeeded by a gradual transformation to the quasi-particle mode, as seen from the figure. The characteristic time for this quasi-particle formation has the order $\hbar /\left(\epsilon_{k}-E_{G}\right)$. The energy entering this estimate is simply the energy distance from the nearest critical point of the density of states. In the present case, it yields a few, like 3, femtoseconds. Over this basic formative process, there is superimposed a much faster and rapidly damped evolution, with a characteristic time on the order of $10^{-1} \mathrm{fs}$. We enclose these details into small windows which are then blown up as inserts. The exact curves in the inserts are fitted by the lowest order cumulant approximations rather well. This testifies explicitly that the gross features of the whole band, as reflected in the lowest moments of the DOS are decisive here. In particular, it is interesting to inspect

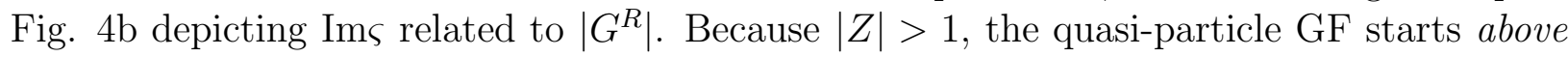
the true one. Nevertheless, the latter quantity starts by a rapid decrease due to a spreading of the probability amplitude into the whole band. Only in the second stage of the formation, a recuperation takes place, and the exact Ims starts to oscillate around the quasi-particle straight line. It is thus not easy in the present case to construct an interpolation form for the GF similar to that introduced in [26]. Instead, we proceed as follows.

The averaged dark GF obeys the Dyson equation in the differential form,

$$
\mathrm{i} \hbar \partial_{t} G^{R}(t)=\epsilon_{\mathrm{MF}} G^{R}(t)+\int \mathrm{d} \bar{t} \Sigma^{R}(t-\bar{t}) G^{R}(\bar{t})
$$

Instead of this integral term involving the self-energy, we may define, in analogy to the Weisskopf-Wigner approximation, a multiplicative, single-time but $k$-dependent, quantity $\sigma_{k}(t)$ by 


$$
\mathrm{i} \hbar \partial_{t} G^{R}(t)=\epsilon_{\mathrm{MF}} G^{R}(t)+\sigma_{k}(t) G^{R}(t)
$$

An explicit "phase" integral equation relating the phase $\varsigma$ with $\Sigma^{R}$ may be developed, but we will not pursue this task.

Comparing with (29), we get the relation

$$
\sigma_{k}(t)=\frac{\mathrm{d}}{\mathrm{d} t} \varsigma(t)
$$

The last three equations combined will be used in SecIVB4 to separate the dark and the induced parts of the self-energy in an efficient manner. This will be based on the short time behavior of $\sigma_{k}(t)$. As plotted in the other panels inserted in Figs. 国a, b, this effective self-energy varies strongly for very short times, but it tends quite rapidly, although in an oscillatory manner, to the constant value of the complex quasi-particle energy.

\section{ILLUMINATED ALLOY}

The electron response to an incident light pulse is conveniently expressed in terms of the non-equilibrium Green function. The full formalism of the NGF is given in references [1] or [2]. Here, only the directly important relations will be given. Our approach is characterized by two features.

(i) We employ the NGF in the real time domain in the Langreth-Wilkins representation [16], that is we work with $G^{R}, G^{<}, G^{A}$. We prefer the LW triplet over the usual KadanoffBaym pair $G^{<}, G^{>}$, because in the present case of a purely elastic scattering the dynamics of the propagators $G^{R}, G^{A}$ becomes decoupled from the particle correlation function $G^{<}$, and is much easier for numerical solution. The LW equations also have a slightly more "physical" appearance, suitable for interpretation.

(ii) For non-interacting electrons, time evolution is strictly unitary even in a disordered system, while the genuine field theoretic description relates to configuration averages [13], [27]. The non-equilibrium Green function needed here can be expressed in terms of configuration averages in a direct fashion following $[8]$.

Below, we describe the LW formalism for the disordered alloys and the computational procedures for the electron propagator reacting to the light pulse, and for the particle correlation function.

\section{A. Non-equilibrium Green functions}

\section{General relations and $L W$ conventions}

We work in the real time domain and the NGF is given by a $2 \times 2$ matrix $\left\|G_{\alpha, \beta}\right\|$, which we choose to be in the Langreth-Wilkins representation [16]:

$$
\mathbf{G}=\left\|\begin{array}{cc}
G^{R} & G^{<} \\
0 & G^{A}
\end{array}\right\|
$$


Here, the component Green functions are one particle operators depending on two time arguments and satisfying the general relations

$$
\begin{aligned}
G^{R}\left(t, t^{\prime}\right) & =\left\{G^{A}\right\}^{\dagger}\left(t^{\prime}, t\right) \\
G^{<}\left(t, t^{\prime}\right) & =-\left\{G^{<}\right\}^{\dagger}\left(t^{\prime}, t\right) \\
G^{>}\left(t, t^{\prime}\right) & =-\left\{G^{>}\right\}^{\dagger}\left(t^{\prime}, t\right) \\
G^{R}-G^{A} & =G^{>}-G^{<}
\end{aligned}
$$

The symmetry relation (42) reduces the number of independent Green functions to two, while the last relation, Eq. (45) (spectral identity), links the LW representation with the usual Kadanoff-Baym pair $G^{<}, G^{>}$.

The so-called LW algebra for the individual components of (41) follows easily from the usual rules for matrix operations, For example, introducing also the self-energy matrix $\Sigma$, we have

$$
\Sigma \mathbf{G}=\left\|\begin{array}{cc}
\Sigma^{R} & \Sigma^{<} \\
0 & \Sigma^{A}
\end{array}\right\| \cdot \| \begin{array}{cc}
G^{R} & G^{<} \\
0 & G^{A}
\end{array}
$$

Thus,

$$
\begin{aligned}
& \{\boldsymbol{\Sigma} \mathbf{G}\}^{R}=\Sigma^{R} G^{R} \\
& \{\Sigma \mathbf{G}\}^{<}=\Sigma^{R} G^{<}+\Sigma^{<} G^{A}
\end{aligned}
$$

We use the convention that all quantities with no time variables shown are double time. Thus, $\boldsymbol{\Sigma} \rightleftharpoons \Sigma_{\alpha, \beta} \equiv \Sigma^{X}\left(t, t^{\prime}\right), H_{\mathrm{MF}} \rightleftharpoons H_{\mathrm{MF}}(t) \delta\left(t-t^{\prime}\right)$, etc. Multiplications mean an operator multiplication and a time integral, like $\Sigma^{R} G^{R} \rightleftharpoons \int \mathrm{d} \bar{t} \Sigma^{R}(t, \bar{t}) G^{R}\left(\bar{t}, t^{\prime}\right)$.

\section{Single alloy configuration}

For non-interacting electrons, time evolution is strictly unitary even in a disordered system: Each alloy configuration gives rise to a random Hermitian one-electron Hamiltonian $\mathcal{H}(t)$, which depends on time due to the effect of external fields (light pulse). The nonrandom configuration independent or averaged quantities are denoted by italics, while the configuration dependent quantities are denoted by rounded characters.

For one configuration, we introduce the usual evolution operator $\mathcal{S}\left(t, t^{\prime}\right)$ corresponding to $\mathcal{H}(t)$, and the one electron density matrix $\varrho(t)$. Selecting the earliest admissible time $t_{0}$, we introduce $\rho\left(t_{0}\right)$ as the initial condition for the NGF. Then, for all times $t, t^{\prime} \geq t_{0}$, the random single configuration NGF is

$$
\mathcal{G}\left(t, t^{\prime}\right)=\left\|\begin{array}{cc}
\frac{1}{\mathrm{i} \hbar} \mathcal{S}\left(t, t^{\prime}\right) \vartheta\left(t-t^{\prime}\right) & -\frac{1}{\mathrm{i} \hbar} \mathcal{S}\left(t, t_{0}\right) \varrho\left(t_{0}\right) \mathcal{S}\left(t_{0}, t^{\prime}\right) \\
0 & -\frac{1}{\mathrm{i} \hbar} \mathcal{S}\left(t, t^{\prime}\right) \vartheta\left(t^{\prime}-t\right)
\end{array}\right\|
$$

Two properties of $\mathcal{G}$ are very important: First, the propagators $\mathcal{G}^{R}, \mathcal{G}^{A}$ do not depend on the particle distribution, and, for propagation from $t^{\prime}$ to $t$, they only depend on $\mathcal{H}(\bar{t})$ with $\bar{t}$ between the two times. Second, the correlation function $\mathcal{G}^{<}$does in fact not depend on the choice of the "initial time" $t_{0}$, if the relation $\varrho(t)=\mathcal{S}\left(t, t_{0}\right) \varrho\left(t_{0}\right) \mathcal{S}\left(t_{0}, t\right)$ is employed. 


\section{Configuration average of $\mathcal{G}$}

Observable quantities are presumably given by the configuration average of the particle distribution function $\rho=\langle\varrho\rangle$ ( see Sec. VI, however); to obtain this quantity, we introduce the averaged non-equilibrium Green function starting from the Eq. (49):

$$
\mathrm{G}=\langle\mathcal{G}\rangle
$$

These configuration averages ought to be treated by field theoretical means [13], [27], [8].

The two propagator components are simple, for $X=R, A$,

$$
G^{X}\left(t, t^{\prime}\right)=\left\langle\mathcal{G}^{X}\left(t, t^{\prime}\right)\right\rangle= \pm \frac{1}{\mathrm{i} \hbar}\left\langle\mathcal{S}\left(t, t^{\prime}\right)\right\rangle \vartheta\left( \pm\left[t-t^{\prime}\right]\right)
$$

Now, we obtain a semi-explicit expression for

$$
G^{<}=\left\langle\mathcal{G}^{<}\right\rangle=\mathrm{i} \hbar\left\langle\mathcal{G}^{R}{ }_{o} \rho_{o} \mathcal{G}^{A}\right\rangle \quad\left(t \geq t_{0}, t^{\prime} \geq t_{0}\right)
$$

Here enters the initial condition at $t=t_{0}$; the initial one-electron distribution is represented in the double time form as

$$
{ }_{o} \rho_{o}=\rho\left(t_{0}\right) \delta\left(t-t_{0}\right) \delta\left(t^{\prime}-t_{0}\right)
$$

This initial condition can be random, that is configuration dependent. We will exclude this possibility in our model by hypothesis. In this paper, we consider the case that the initial distribution $\rho\left(t_{0}\right)$ is non-random. By this, we eliminate the problem of initial correlations [28]. For the non-random initial condition, the averaged product in (52) can be written as

$$
G^{<}=\underbrace{\mathrm{i} \hbar G^{R} \rho_{o} G^{A}}_{\text {COHERENT }}+\underbrace{G^{R} \Sigma^{<} G^{A}}_{\text {INCOHERENT }}
$$

This equation defines $\Sigma^{<}$, which is a regular two-time kernel in this case. The Eq. (54) is then nothing else than the <-component of the Dyson equation for the non-equilibrium Green function matrix (41). It is a good example of the suggestive LW form of the theory. It has the full $R \leftrightarrow A$ symmetry and a causal structure. The initial state evolves coherently in resemblance to unaveraged $\mathcal{G}^{<}$(cf. Eq. (52)), but it is evanescent. The population is replenished by an incoherent backscattering due to $\Sigma^{<}$. Eq. (54) is also a starting point for developing equations of the Bethe-Salpeter type and the quantum transport equations.

Given these results, we introduce the corresponding Dyson equation in the differential form by starting from the equations for the unaveraged Green function and performing the configuration average term by term.

The random Hamiltonian can be divided into its configuration average, the mean field Hamiltonian, and the fluctuation potential, that is the configuration dependent random field: $\mathcal{H}(t)=H_{\mathrm{MF}}(t)+\mathcal{D}(t)$, as introduced by Eq. (9). Both parts of $\mathcal{H}(t)$ might be time dependent at this level of generality, in contrast to the specific model, Eqs. (12), (13) which we employ for explicit calculations in this paper.

By the Eqs. (51), (52), and (9), 


$$
\begin{aligned}
\left(\mathrm{i} \hbar \partial_{t}-H_{\mathrm{MF}}\right) \mathcal{G}-\mathcal{D} \mathcal{G} & =\boldsymbol{I} \\
\left(\mathrm{i} \hbar \partial_{t}-H_{\mathrm{MF}}\right) \mathbf{G}-\boldsymbol{\Sigma} \mathbf{G} & =\boldsymbol{I}
\end{aligned}
$$

Here, $\partial_{t} \rightleftharpoons \frac{\partial}{\partial t} \delta\left(t-t^{\prime}\right), \boldsymbol{I} \rightleftharpoons \delta_{\alpha, \beta} I \rightleftharpoons \delta_{\alpha, \beta} 1_{\mathrm{OP}} \delta\left(t-t^{\prime}\right)$, and $\mathcal{D} \rightleftharpoons \mathcal{D}(t) \boldsymbol{I}$. On going from (55) to (56), we define the self-energy matrix by setting $\langle\mathcal{D} \mathcal{G}\rangle \equiv \boldsymbol{\Sigma} \mathbf{G}$. The possibility of such definition is itself another form of saying that the initial condition is non-random. A brief discussion of the random correlated initial condition is deferred to the Appendix $\mathrm{A}$.

The Dyson equation (56) has to be supplemented by an actual form of the self-energy in order to become closed. In this paper, we will use the Self-consistent Born approximation (SCBA), which is a generic self-consistent conserving approximation appearing as a leading term either in the self-consistent multiple scattering expansion, or in the self-consistent perturbation expansion. The relationship between SCBA and the Coherent potential approximation within the multiple scattering context is discussed in [8]. Here, we use the self-consistent perturbation approach. This is based on the integral equations

$$
\begin{aligned}
& \mathcal{G}=\mathrm{G}+\mathrm{G}(\mathcal{D}-\boldsymbol{\Sigma}) \mathcal{G} \\
& \mathcal{G}=\mathrm{G}+\mathcal{G}(\mathcal{D}-\Sigma) \mathrm{G}
\end{aligned}
$$

which follow from Eqs. (55), (56), and which can be iterated to yield a renormalized Born series in terms of the full propagators $\mathcal{G}$ and the renormalized perturbation $\mathcal{D}-\boldsymbol{\Sigma}$. To obtain a closed equation for the self-energy, we iterate (57) once using (58) and configuration average respecting Eq. (111):

$$
\Sigma=\langle\mathcal{D} \mathcal{G} \mathcal{D}\rangle-\Sigma \mathrm{G} \Sigma
$$

This yields an iterative expansion in terms of $\mathcal{D}$ and $\mathcal{G}$

$$
\begin{aligned}
& \Sigma=\overbrace{\langle\mathcal{D G} \mathcal{D}\rangle}^{\Sigma_{\mathrm{SCBA}}}+\langle\mathcal{D G} \mathcal{D G} \mathcal{D}\rangle+ \\
& +\langle\mathcal{D G} \mathcal{D} G \mathcal{D} G \mathcal{D}\rangle-\langle\mathcal{D} G \mathcal{D}\rangle \mathrm{G}\langle\mathcal{D} \mathrm{G} \mathcal{D}\rangle-\langle\mathcal{D} \mathrm{G}\langle\mathcal{D} \mathrm{G} \mathcal{D}\rangle \mathrm{G} \mathcal{D}\rangle+\ldots
\end{aligned}
$$

The expansion starts from the second order in $\mathcal{D}$, which is our SCBA. In the fourth order, the expansion already deviates from a simple geometrical series. In the language of diagrams, the first subtraction excludes a reducible diagram, the second suppresses double counting of self-energy insertions in the inner propagator lines [13]. The physical approximation for $\boldsymbol{\Sigma}$, like a termination of the expansion at some order, is made consistently for all components of the self-energy, which guarantees its self-consistent conserving property. This is also the case of the SCBA.

\section{B. Working form of the Dyson equations}

Now we write explicitly the equations for all components of the NGF in the form suited for numerical solution. These are obtained as the corresponding components of the matrix equations (56) and (50) with the use of the LW rules (46) - (48). For each component, we 
have to specify: $\diamond$ the equation of motion; $\diamond$ initial conditions; $\diamond$ the specific form of the SCBA self-energy.

We repeat that the equations will be written in the Galitskii picture with the convention that

the operators, Green functions, etc., will be written without tildes. To return to the Schrödinger picture, we will use the subscript ...s.

\section{Explicit equations for $\boldsymbol{G}^{\boldsymbol{R}}, \boldsymbol{G}^{<}$}

For the propagators, we get

$$
\begin{gathered}
\left(\mathrm{i} \hbar \partial_{t}-H_{\mathrm{MF}}\right) G^{R, A}-\Sigma^{R, A} G^{R, A}=I \\
G^{R}\left(t=t^{\prime}+0, t^{\prime}\right)=\mathrm{i} \hbar^{-1} \cdot 1_{\mathrm{OP}} \\
\Sigma_{\mathrm{SCBA}}^{R, A}=\left\langle\mathcal{D} G^{R, A} \mathcal{D}\right\rangle
\end{gathered}
$$

In fact, only one of the propagators has to be found directly, as the other one is given by (42).

For the particle correlation function, the three relations are as follows

$\diamond \quad$ The equation of motion has no $\delta$-singularity:

$$
\left(\mathrm{i} \hbar \partial_{t}-H_{\mathrm{MF}}\right) G^{<}=\Sigma^{R} G^{<}+\Sigma^{<} G^{A}
$$

$\diamond$ It has to be integrated for all times $t>t_{0}$ for any fixed $t^{\prime} \geq t_{0}$. Thus, the initial value is $G^{<}\left(t_{0}, t^{\prime}\right)$. For a non-random initial distribution $\rho\left(t_{0}\right)$, we get immediately

$$
G^{<}\left(t_{0}, t^{\prime}\right)=\rho\left(t_{0}\right) G^{A}\left(t_{0}, t^{\prime}\right)
$$

$\diamond$ The SCBA self-energy $\Sigma^{<}$has a form analogous to the propagator component:

$$
\Sigma_{\mathrm{SCBA}}^{<}=\left\langle\mathcal{D} G^{<} \mathcal{D}\right\rangle
$$

We will return to the equations (61) - (66) below and show the methods of their practical handling and the computed electron dynamics. Before that, we will make our model fully explicit.

First, we select the initial distribution function by assuming that the valence band is completely occupied, the conduction band entirely empty:

$$
\rho\left(t_{0}\right)=\sum_{\mathrm{k} \in \mathrm{BZ}}|v \mathrm{k}\rangle\langle v \mathrm{k}| \equiv P_{v}
$$

As indicated, the $\rho\left(t_{0}\right)$ operator coincides with the projector on the valence band states, which is non-random and alloy composition independent by assumption, so that (65) applies.

Second, all equations have to be specialized for the two band model of Sec.IIB. Thus, all GF, etc., will be $k$-diagonal 2x2 matrices: $G^{X} \rightleftharpoons\left\|G_{a b}^{X}\left(k ; t, t^{\prime}\right)\right\|,\{X=R, A,<; a, b=$ 
$c, v ; k \in \mathrm{BZ}\}$ and similarly for the self-energy. Disorder is acting only in the conduction band, so that only the $c c$ components of the self-energy are non-zero. The self-energy becomes $k$-independent in the single-site approximation. By this, the general relation (63) simplifies to a scalar equation in the spirit of the dark relations (17):

$$
\begin{aligned}
\Sigma_{c c}^{X}\left(t, t^{\prime}\right) & =\gamma \cdot F_{c c}^{X}\left(t, t^{\prime}\right) \\
F_{c c}^{X}\left(t, t^{\prime}\right) & =N^{-1} \sum_{k \in \mathrm{BZ}} G_{c c}^{X}\left(k ; t, t^{\prime}\right) \\
\gamma & =c(1-c) \delta^{2}
\end{aligned}
$$

\section{Separation of the dark self-energy}

In principle, we have to solve the Dyson equations (61) for $G^{R}$ and (64) for $G^{<}$, and to simultaneously obtain the self-energies from the self-consistent relations (63), (66). These equations will first be modified, however, by singling out the dark constituent of the selfenergy. For these modified equations, a self-consistent cycle leads to their stable solution. The self-energy will be divided into its dark and induced components not only for computational, but also for physical reasons. The dark component analyzed in the preceding section describes the polaron effects including the short time dynamics and the long time quasi-particle behavior. The induced component reflects the coherent coupling between the excitation and the scattering. Proper inclusion of this coherence effect appears to be essential to keep the theory conserving and consistent, as is documented below.

We will separate out the dark part of the retarded self-energy; what remains, is the induced part:

$$
\Sigma^{R}=\Sigma_{\mathrm{DARK}}^{R}+\Sigma_{\mathrm{INDUCED}}^{R}
$$

No such separation is needed for the particle component, because all of it is induced in our case:

$$
\Sigma^{<}=\Sigma_{\text {INDUCED }}^{<}
$$

For convenience, the subscripts will be shortened to D and I.

The equations (61) and (63) for the retarded propagator become:

$$
\begin{aligned}
\mathrm{i} \hbar \partial_{t} G^{R} & -\overbrace{\left(H_{\mathrm{MF}}+\Sigma_{\mathrm{D}}^{R}\right)}^{H_{\mathrm{QP}}} G^{R}=\Sigma_{\mathrm{I}}^{R} G^{R}, \quad t>t^{\prime} \\
\Sigma_{\mathrm{I}}^{R} & =\left\langle\mathcal{D}\left(G^{R}-G_{\mathrm{D}}^{R}\right) \mathcal{D}\right\rangle
\end{aligned}
$$

The dark self-energy is known once for ever for a given system, and it appears as a permanent renormalisation ("polaron shift") at the 1.h.s. of the Dyson equation. There is no coupling between this renormalizaton and the light. Without $\Sigma_{\mathrm{I}}^{R}$, the pulse would act as if it were exciting preexisting dark quasi-particles. Furthermore, the dark self-energy acts on the 
propagator almost as if it were local in time. For all these reasons, we denote the operator on the lhs of the Dyson equation by $H_{\mathrm{QP}} \ldots$ an effective quasi-particle Hamiltonian.

Only the Dyson equation (64) is modified for $G^{<}$in view of Eq. (70),

$$
\mathrm{i} \hbar \partial_{t} G^{<}-\overbrace{\left(H_{\mathrm{MF}}+\Sigma_{\mathrm{D}}^{R}\right)}^{H_{\mathrm{QP}}} G^{<}=\Sigma_{\mathrm{IND}}^{R} G^{<}+\Sigma^{<} G^{A} \quad t>t_{0}
$$

while the equations (66), (65) remain without change. This triplet Dyson equation-SCBAinitial condition is similar in many respects to the system for the retarded component. In particular, the lhs of the Dyson equation has again the "polaron" interpretation of dark quasiparticles excited by the light pulse.

\section{Induced part of the retarded self-energy}

To see in better detail the structure of the induced part of the self-energy, we rewrite it explicitly:

$$
\Sigma_{c c, \mathrm{I}}^{R}\left(t, t^{\prime}\right)=\gamma N^{-1} \sum_{\mathrm{k} \in \mathrm{BZ}}\left(G_{c c}^{R}\left(\mathrm{k} ; t, t^{\prime}\right)-G_{c c, \mathrm{D}}^{R}\left(\mathrm{k} ; t, t^{\prime}\right)\right)
$$

Three points are apparent.

First, $\Sigma_{\mathrm{I}}^{R}\left(t, t^{\prime}\right)=0$, if the interval $\left[t, t^{\prime}\right]$ has no overlap with the time span of the pulse, because then $G^{R}=G_{\mathrm{D}}^{R}$. Thus $\Sigma_{\mathrm{I}}^{R}$ vanishes in the quiescent time domain, and need not be computed for these times. This SCBA result coincides with its exact counterpart. Namely, in an exact treatment, the Green function is essentially a configuration average of a unitary evolution operator. The evolution is governed by a time local Hamiltonian, and the configuration average is also instantaneous. As a result, the averaged propagation from $t^{\prime}$ to $t$ cannot depend on external fields acting outside this interval, just like in the approximate treatment.

Second, the excitation by a pulse is not homogeneous in the whole Brillouin zone, acting predominantly in a strip of a width $\sim Q$ around the resonant energy. In the parts of the BZ far from this strip, the excitation is weak, and the full Green function deviates but weakly from the dark one. This averts the necessity to integrate over the whole zone - in contrast to the dark self-energy itself. Thus, only the physically relevant $k$-vectors are involved, and the details of the distant parts of the band structure have only a minor importance.

This, in turn, reduces further the time domain, where the induced self-energy deviates from zero. While it vanishes exactly for initial times $t^{\prime}$ after the pulse, we may conclude now that it is also negligible for $t^{\prime}$ well before the pulse. Namely, both $G^{R}$ and $G_{\mathrm{D}}^{R}$ are exponentially damped at a rate roughly corresponding to the resonant energy for all relevant $\mathrm{k}$; the whole induced self-energy is thus exponentially small in $t-t^{\prime}$ as $t$ reaches the time of the pulse.

Third, the induced part $\Sigma_{\mathrm{I}}^{R}$ is continuous for equal times, $t=t^{\prime}$. Each of the self-energies has a $\gamma / \mathrm{i} \hbar$ jump at equal times, and these two jumps exactly compensate. By extending the moment analysis of Sec. [IIC also to the full $G^{R}$, it is possible to improve the short time 
estimate of $\Sigma_{\mathrm{I}}^{R}$. The moments depend on the inital time $t^{\prime}$, and we quote without details that Eq. (37) is generalized to

$$
\begin{aligned}
G_{c c}^{R}\left(\mathrm{k} ; t, t^{\prime}\right) \approx & \frac{1}{\mathrm{i} \hbar} \mathrm{e}^{-\frac{\mathrm{i}}{\hbar} \epsilon_{\mathrm{MF}}(\mathrm{k})\left(t-t^{\prime}\right)} \times \\
& \left(1-\left(\gamma+Q^{2} \phi^{2}\left(t^{\prime}\right)\right) \frac{\left(t-t^{\prime}\right)^{2}}{\hbar^{2}}-\ldots\right) \\
\approx & \frac{1}{\mathrm{i} \hbar} \mathrm{e}^{-\frac{\mathrm{i}}{\hbar} \epsilon_{\mathrm{MF}}(\mathrm{k})\left(t-t^{\prime}\right)} \times \mathrm{e}^{-\left(\gamma+Q^{2} \phi^{2}\left(t^{\prime}\right)\right) \frac{\left(t-t^{\prime}\right)^{2}}{\hbar^{2}}}
\end{aligned}
$$

For the shortest times, the contributions to the "action" due to light and to the disorder are independent and additive. Introducing this expansion into the Eq. (73), we deduce the short time behavior of $\Sigma_{\mathrm{I}}^{R}$ in the form

$$
\left.\Sigma_{c c, \mathrm{I}}^{R}\left(t, t^{\prime}\right)=\gamma Q^{2} \phi^{2}\left(t^{\prime}\right)\right) \mathrm{e}^{-\frac{\mathrm{i}}{\hbar}\langle\epsilon\rangle\left(t-t^{\prime}\right)} F_{o}\left(t-t^{\prime}\right) \frac{\left(t-t^{\prime}\right)^{2}}{\hbar^{2}}+\mathcal{O}\left(\left(t-t^{\prime}\right)^{3}\right)
$$

Here, $F_{o}(t)$ is the Fourier transform of the pure crystal local Green function, see (18). This is an important result, because it shows that the complicated short time behavior of the dark Green function does not enter the induced part of the self-energy, which gives it a welcome robustness with respect to approximations for Green functions.

\section{Dark quasi-particles}

So far, the definition $H_{\mathrm{MF}}+\Sigma_{\mathrm{D}}^{R}=H_{\mathrm{QP}}$ had only a symbolic meaning, and was not associated with an explicit introduction of the quasi-particle picture into the computations. There are reasons, both practical and theoretical, to investigate this possibility in more detail. On the practical side, the dark self-energy varies strongly in a very short time interval. To include it properly means to perform the integrations on the lhs of the Dyson equation with a great care. At the same time, it is clear that beyond a short formation time, the dark propagators will assume the Weisskopf-Wigner form of a renormalized time exponential. Instead of directly using these WW propagators as a basis, we recall their phase form discussed in Sec. III . The GF of that section coincides with $G_{c c}^{R}$, the time $t$ is replaced by $t-t^{\prime}$ in the non-stationary situation in the Eq. (39). For large time differences, the action of $\Sigma_{\mathrm{D}}^{R}$ will approach the WW limit as given by $(\overline{21}), \sigma_{k}(t) \longrightarrow \sigma_{k}(\infty)=\gamma \cdot F_{o}\left(\epsilon_{k} \pm \mathrm{i} 0\right)$ regardless of the presence of the external field. On the other hand, for short times $t-t^{\prime}$, we have shown in Eq. (74) that the phase variations caused by disorder and by the light are additive. Thus, the effect of the disorder and of the external disturbance appear as additive both in the short time and in the long time limits. Interpolating this behavior, we arrive at the following quasi-particle approximation:

$$
\left.H_{\mathrm{QP}}\right|_{k}=\left\|\begin{array}{cc}
\epsilon_{c}(k)+\langle\epsilon\rangle+\sigma_{k}\left(t-t^{\prime}\right) & -Q \Phi(t) \\
-Q \Phi(t) & \epsilon_{v}(k)+\hbar \Omega
\end{array}\right\|
$$

It is understood that this effective Hamiltonian acts on a Green function with time arguments $t, t^{\prime}$ and $\sigma_{k}\left(t-t^{\prime}\right)$ acts as a multiplicative quantity. If the renormalization effects are not strong (slow energy variation of $F_{0}$ ), a further approximation, pure WW $\sigma_{k}\left(t-t^{\prime}\right) \approx \sigma_{k}(\infty)$, may be tested, as we did in [1]. 


\section{Computational schemes}

\section{Computational scheme for the propagator}

We recall once more the important point that the propagator dynamics is fully independent of the particle distribution in our model with elastic scattering. The propagators and the related self-energies can thus be computed from Eq. (71) in an independent round of the whole calculation. This is a comparatively less demanding computational task. Only the retarded GF and self-energy need to be obtained in a self-consistent cycle. The results can be stored, the advanced counterparts are obtained using the symmetry (42): $G^{A}\left(t, t^{\prime}\right)=\left[G^{R}\left(t^{\prime}, t\right)\right]^{\dagger}, \Sigma^{A}\left(t, t^{\prime}\right)=\left[\Sigma^{R}\left(t^{\prime}, t\right)\right]^{\dagger}$. In Fig. 5, we show the flowchart for computing $\Sigma_{\mathrm{I}}^{R}$. For solving the Dyson equation, we used a fourth order adaptive RungeKutta-Fehlenberg solver. Interesting is the seemingly counterintuitive order of time loops. This is dictated by the fact that we are bound to make one step in the floating time, $t \longrightarrow t+\Delta t$, for all intermediate initial times $t>\bar{t}>t^{\prime}$, so as to be able to calculate the product $\Sigma_{\mathrm{I}}^{R} G^{R} \longrightarrow \int \mathrm{d} \bar{t} \Sigma_{\mathrm{I}}^{R}(t+\Delta t, \bar{t}) G^{R}\left(\bar{t}, t^{\prime}\right)$ for the new time $t+\Delta t$.

\section{Computational scheme for the particle function}

On the whole, the Eqs. (71) and (72) have an analogous structure. There are also marked differences. For $G^{<}$, the integration of the Dyson equation starts at $t=t_{0}$ for any fixed $t^{\prime}$. The advanced propagator enters (72) at two places, in the initial condition and on the rhs of the Dyson equation in the integral $\int \mathrm{d} \bar{t} \Sigma^{<}(t, \bar{t}) G^{A}\left(\bar{t}, t^{\prime}\right)$. Thus, $G^{A}\left(\bar{t}, t^{\prime}\right)$ is needed for $\left.t_{0} \leq \bar{t} \leq t^{\prime}\right)$. It is readily available from the retarded propagator $G^{R}\left(t^{\prime}, \bar{t}\right)$ calculated and stored beforehand, at the retarded stage, by means of the crossing symmetry (42). Another difference is that the self-consistent equations for $G^{<}$and $\Sigma^{<}$are linear, once the propagators are known, so that no iteration for obtaining $\Sigma^{<}$is needed in principle, and the integration process runs only once. An iteration of the process appears as necessary on the practical level, however. Namely, in contrast to the retarded case, where $\Sigma^{R}(t, t)=0$ is known, there is no universal value of $\Sigma^{<}(t, t)$, and this quantity must be estimated by extrapolation and refined by iteration. The whole process is outlined in a flow chart shown in Fig. 6. For the particular Hamiltonian (8) and $\mathcal{D}$ given by (13), the explicit form of the integral for $\Sigma^{<}$is

$$
\begin{gathered}
\Sigma_{c c}^{<}\left(t, t^{\prime}\right)=\gamma N^{-1} \sum_{\mathrm{k} \in \mathrm{BZ}} G_{c c}^{<}\left(\mathrm{k} ; t, t^{\prime}\right) \\
\gamma=c(1-c) \delta^{2}
\end{gathered}
$$

The number of primitive cells is denoted by $N$, the Nordheim parameter $\gamma$ as the second cumulant of $\mathcal{D}$ measures the disorder scattering strength in the SCBA. No subtraction of the dark GF is needed, because without illumination the electrons cannot leave the valence band, $G_{\mathrm{D}}^{<}=P_{v} G_{\mathrm{D}}^{<} P_{v}$, while disorder is confined to the conduction band. All of the $c c$ component of the full $G^{<}$is induced, as stated in Eq. (70). The integration in (77) extends in fact only over a narrow shell around the one-photon resonance, just like in the integral (71) for $\Sigma_{\mathrm{IND}}^{R}$. There, however, the subtraction is crucial. 


\section{NUMERICAL EXAMPLE}

In this section, we show a representative numerical example for the alloy model defined in Sections $\llbracket \mathrm{IB}$ and $\amalg \Pi \mathrm{B}$. The bare A-crystal band structure is characterized by the band gap $E_{G}=1.5 \mathrm{eV}$, the electron and hole masses $m_{c}=0.4 m_{e}, m_{v}=0.6 m_{e}$. The bands are "proportional", with band widths $12 \mathrm{eV}$, and $8 \mathrm{eV}$, respectively. The band density of states is given in IIIB. The alloy parameters are: $c=0.05, \delta=-0.84 \mathrm{eV}$, so that $\langle\epsilon\rangle=0.042 \mathrm{eV}$ and $\gamma=0.034 \mathrm{eV}^{2}$. We use the basic frequency $\hbar \Omega=2 \mathrm{eV}$ for a pure A-crystal and adjust the frequency for the actual $c$ to $1.75 \mathrm{eV}$ according to Table [ The exciting light pulse is specified in $\mathrm{VA}$ below.

All parameters are selected so that they lead to pronounced but moderate effects. The formation times are shorter than either the pulse duration or the electron relaxation time, and the photoexcited electron density is sufficiently low that the $e-e$ relaxation time is longer than both these times.

\section{A. Pulse parameters}

We have to specify the pulse strength $Q$ and its shape $\Phi(t)$, see (6). The pulse strength is $Q=\frac{1}{2} e \mathrm{E}_{\mathrm{M}}\langle c \mathrm{k} \simeq 0|(\mathrm{e}, \mathrm{r})| v \mathrm{k} \simeq 0\rangle$ by (6). The $x$-matrix element is estimated as the lattice spacing $a_{0} \approx 10$ a.u., corresponding to the momentum matrix element of about 0.5 a.u. As $\max \Phi=1$, we get the estimate $\mathrm{E}_{\mathrm{M}}\left[\mathrm{Vm}^{-1}\right]=1.89 \times 10^{9} Q[\mathrm{eV}]$ for the peak value of the electric field.

We use the "sech" shape of the pulse and control its duration by a single parameter $t_{\mathrm{P}}$ :

$$
\begin{aligned}
& \Phi(t)=\varphi\left(\left(t-t_{1 / 2}\right) / t_{\mathrm{P}}\right) \\
& \varphi(t)=4 /\left(\mathrm{e}^{t}+\mathrm{e}^{-t}\right)^{2}
\end{aligned}
$$

The $t_{1 / 2}$ shift is defined by the condition $\Phi(0)=\frac{1}{2}$. This shift and the full width at the half maximum of intensity are related to $t_{\mathrm{P}}$ by 29

$$
\begin{aligned}
t_{1 / 2} & =t_{\mathrm{P}} \cdot 0.88137 \\
t_{\mathrm{FWHM}} & =t_{\mathrm{P}} \cdot 1.21169
\end{aligned}
$$

A convenient integral measure of the pulse is the resonant Rabi phase,

$$
\varphi_{\mathrm{R}}=\int_{-\infty}^{+\infty} \mathrm{d} t \Omega_{\mathrm{R}}(t)
$$

where the Rabi frequency is introduced by

$$
\frac{1}{2} \hbar \Omega_{\mathrm{R}}(t)=Q \phi(t)
$$

For our pulse shape (78) this gives

$$
\begin{aligned}
\varphi_{\mathrm{R}} & =4 \hbar^{-1} t_{\mathrm{P}} Q \\
& =3.3012 \hbar^{-1} t_{\mathrm{FWHM}} Q
\end{aligned}
$$

We use a pulse with characteristics according to the Table [1]. 


\section{B. Propagators}

\section{Calculated $\boldsymbol{\Sigma}_{\mathrm{I}}^{R}\left(t, t^{\prime}\right)$}

$G^{R}$ and $\Sigma^{R}$ are given by the Eqs. (71) and (73). These were solved as explained in IVQ. First, we present in Fig. 7 the imaginary (dominant) part of the induced self-energy as a function of both times $t, t^{\prime}$. This calculated shape manifests the properties predicted in Subsec. IVB3. As a function of $t$ for a fixed initial time $t^{\prime}$, it is strictly zero for $t<t^{\prime}$ and it continuously assumes non-negative values on crossing the diagonal $t=t^{\prime}$. For $t>t^{\prime}$, it shows a marked memory effect, that is time non-locality, or temporal coherence, and it ends with a tail corresponding to the dark decay rate of the excitation after the end of the pulse. In the perpendicular direction, as a function of $t^{\prime}$ for $t$ fixed, the $\operatorname{Im} \Sigma^{R}$ I profile is symmetric, decaying exponentially for times $t^{\prime}$ well before the pulse arrival and ending continuously but abruptly at the upper limit. Across the time diagonal, the damping is much faster, as seen both in the main plot, and in the rotated insert. To motivate this, we note that in the Wigner coordinate $t-t^{\prime}$, the exponential damping has a doubled decrement: $G^{R}$ 's in (73) depend only on $t-t^{\prime}$ once both times are large enough, and for $t+t^{\prime}=$ const, we have $t-t^{\prime}=2 t-$ const. Along the time diagonal, as a function of $t+t^{\prime}, \operatorname{Im} \Sigma_{\mathrm{I}}^{R}$ repeats well the shape of the pulse.

The induced self-energy has a much smaller magnitude than its dark counterpart, but it persists much longer. As a consequence, it is difficult to qualitatively guess the relative importance of both components of the self-energy for the resulting propagators.

\section{Calculated $\boldsymbol{G}^{\boldsymbol{R}}\left(\boldsymbol{t}, \boldsymbol{t}^{\prime}\right)$}

In the two-band model, the propagators are given by $2 \times 2$ complex matrices $\left\|G_{a, b}^{R}\left(\mathrm{k} ; t, t^{\prime}\right)\right\|$. We show the behavior of the GF for just one point in the BZ, namely $\mathrm{k} \approx \mathrm{k}^{\times}$, by examining the time evolution of two quantities, $\left|G_{c c}^{R}\right|^{2}$ and $\left|G_{v v}^{R}\right|^{2}$. For a time development which started from a pure $\mathrm{k}^{\times} c$ - or from a pure $\mathrm{k}^{\times} v$ - state, respectively, they measure the probability that the particle stays in its initial state. In Fig. 8, we plot the two quantities as a function of $t$ and $t^{\prime}$. For equal times, they start from a constant value $\hbar^{-2}$. For initial times well out of the pulse time interval, the decay of the $c$ state resembles the dark alloy, while the $v$ state does not decay at all. Within the pulse region, the very earliest evolution is still dominated by the dark self-energy. Soon, however, the external field controls the evolution, and the Rabi oscillation develops.

This qualitative picture does not change, if the induced part of the self-energy is switched off, as depicted by plotting two surfaces in each panel of the figure, which resemble each other quite closely. The differences are but quantitative. In other words, the dark quasiparticle approximation of Sec. IVB 4 neglecting $\Sigma_{\mathrm{I}}^{R}$ is qualitatively satisfactory. It has its serious problems, however, as we show now. 


\section{Testing the semi-group property}

There exists a sensitive test of the quasi-particle behavior of the retarded Green function, which leads to an easy and convincing proof of the importance of the induced self-energy [11]. The true Weisskopf-Wigner dark quasi-particles, with zero illumination, would have an exponentially damped modulus. A generalization proper for the dark quasi-particle approximation in the WW limit, as defined in Sec. IVB4, employs the time-locality of the WW quasi-particle Hamiltonian to justify the semi-group multiplicative property of the propagator. This is summarized in the following equations:

$$
\begin{gathered}
\mathrm{i} \hbar \mathcal{G}^{R}\left(t, t^{\prime}\right)=\mathrm{i} \hbar \mathcal{G}^{R}\left(t, t^{\prime \prime}\right) \cdot \mathrm{i} \hbar \mathcal{G}^{R}\left(t^{\prime \prime}, t^{\prime}\right) \\
\mathrm{i} \hbar G^{R}\left(t, t^{\prime}\right) \neq \mathrm{i} \hbar G^{R}\left(t, t^{\prime \prime}\right) \cdot \mathrm{i} \hbar G^{R}\left(t^{\prime \prime}, t^{\prime}\right) \\
\mathrm{i} \hbar G_{\mathrm{wW}}^{R}\left(t, t^{\prime}\right)=\mathrm{i} \hbar G_{\mathrm{wW}}^{R}\left(t, t^{\prime \prime}\right) \cdot \mathrm{i} \hbar G_{\mathrm{wW}}^{R}\left(t^{\prime \prime}, t^{\prime}\right) \\
t \geq t^{\prime \prime} \geq t^{\prime}
\end{gathered}
$$

For the unaveraged GF, the equality follows from Eq. (49), while for the exact averaged GF, the inequality expresses the fact that a product of averages does not equal to the average of a product of two quantities. For the quasi-particle case, the equality is restored by the time locality of the effective Hamiltonian (76). We study again $G^{R}$ for the resonant $k$-state starting this time at $t^{\prime}=-0.2 \mathrm{ps}$. It is preferable to examine the squares of the full coherent amplitudes, $\left|G_{c c}^{R}\right|^{2}+\left|G_{v c}^{R}\right|^{2}$ and $\left|G_{v v}^{R}\right|^{2}+\left|G_{c v}^{R}\right|^{2}$. In Fig. 9, we see how the $c$-band state initially decays with the dark decay rate, but later one Rabi flip is apparent. The $v$-band state is stationary at first. The pulse leads to its substantial depletion; a hint of the Rabi oscillation is also noticeable. The left panels incorporating $\Sigma_{\mathrm{I}}^{R}$ and the right panels using the WW quasi-particle approximation lead to a picture qualitatively resembling the exact result to a large degree.

Now we start testing the semi-group property (83). As marked by squares on the profile of the pulse, several instants $t^{\prime \prime}$ were selected, at which we factorized the GF and plotted in various thin lines the time evolution corresponding to $\mathrm{i} \hbar G^{R}\left(t, t^{\prime \prime}\right) \mathrm{i} \hbar G^{R}\left(t^{\prime \prime},-0.2\right)$ for $t>t^{\prime \prime}$. While these thin lines coalesce with the full line of the non-factorized Green function in the quasi-particle case, the factorization for the true GF appears as approximately correct

only for factorization times well before or well after the maximum of the pulse, when, of course, we deal with a nearly dark evolution, so that the time non-locality of the self-energy does not play an important role. We will see in Sec. VIB2, how these deviations from the quasi-particle behavior influence also the evolution of the photo-excited electron population.

It should be pointed out that these results were obtained in the WW limit, because the discrepancy between both cases is particularly clear. Similar differences caused by the neglect of $\Sigma_{\mathrm{I}}^{R}$ appear also in the general case and are not peculiar to the WW approximation.

\section{Particle correlation function}




\section{Calculated $\boldsymbol{\Sigma}^{<}\left(\boldsymbol{t}, \boldsymbol{t}^{\prime}\right)$}

The 'less' component of the self-energy is the central quantity for a full description of the excitation and transport processes generated by the pulse: in the Bethe-Salpeter equation $\Sigma^{<}$appears as an irreducible vertex incorporating all correlations, which are in the present case due to the configuration average of the coherent multiple scattering of an $e$ - $h$ pair out of the equilibrium. As stressed already in Secs. IV B 2 and IV C2, $\Sigma^{<}$would be zero without an excitation to the $c$ band which activates the disorder scattering. $\Sigma^{<}$has a coherent component resembling the pulse shape and width along the time diagonal $t-t^{\prime}=0$, as shown in Fig. 10. This is similar to $\Sigma_{\text {IND }}^{R}$, Fig. 7. A marked new feature, however, is the appearance of a tail of $\Sigma^{<}$which persists beyond the pulse duration. This is connected in a self-consistent manner with the photoexcited population in the $c$ band. For the SCBA, we may see it clearly from the Eq. (77), by which $\Sigma^{<}$is proportional to $\operatorname{Tr} G_{c c}^{<}$.

Finally, we look in the direction of the time diagonal (the arrow in the figure) to inspect the profile of $\Sigma^{<}$along the other, $t+t^{\prime}$, diagonal. In the small inserts, the plots of the real and the imaginary part of the self-energy are correspondingly rotated and the symmetry relations equivalent to (43) are demonstrated. This represents a non-trivial check of the numerical approach used, because the integration procedure based on the differential Dyson equation (72) is not a priori symmetric with respect to both times.

\section{Calculated $G^{<}\left(t, t^{\prime}\right)$}

Fig. 11 shows just one representative $G_{c c}^{<}\left(\mathrm{k} ; t, t^{\prime}\right)$, namely that for k near the one-photon resonance. Symmetries (43) are verified as a feature of individual terms of (77), as shown in the format of Fig. 10. We will concentrate on the even part $\hbar \operatorname{Im} G_{c c}^{<}\left(\mathrm{k} ; t, t^{\prime}\right)$, because its time diagonal equals to the induced population of the $|c \mathrm{k}\rangle$ state. The induced transient is followed by pronounced structures slowly decaying along both time axes $t, t^{\prime}$ and by a slow rise tending to saturation along the time diagonal $t=t^{\prime}$. A closer look shows about one full wave along the diagonal, in agreement with the Rabi phase of our pulse being adjusted to about $2 \pi$. The side wings display only one rise followed by a decrease suggesting a phase variation $\approx \pi$. To understand the origin of this behavior better, we split in Fig. 12 the Green function into its coherent and incoherent parts in accordance with (54). The side arms of $\operatorname{Im} G_{c c}^{<}$appear to originate from its coherent part $\operatorname{Im} G_{\mathrm{COH}}^{<}$. In fact, for one time fixed, the profile along the other time axis is proportional to $G^{R}$, so that its oscillation is given by the Rabi phase halved, and the attenuation at long times is $\hbar / \operatorname{Im} \Sigma_{\text {DARK }}^{R}$. Along the time diagonal, $G_{\mathrm{COH}}^{<}$is bilinear in $G^{R}$, and the attenuation time equals now to the dark quasiparticle lifetime $\hbar / 2 \operatorname{Im} \Sigma_{\text {DARK }}^{R}$ leading to a much faster decay. The incoherent part $\operatorname{Im} G_{\mathrm{INCOH}}^{<}$appears with a time lag due to the multiple scattering delays. It is concentrated to a zone along the time diagonal, where it gradually supersedes the coherent part, so that it is responsible for the final rise of the full $\operatorname{Im} G^{<}$in Fig. 11.

This description is somewhat oversimplified and would be correct only in the dark quasiparticle approximation of TVB4, in which the exponentially decaying quasiparticles are driven by the incident light. In fact, the Eq. (72) goes beyond the quasiparticle picture by incorporating coherence between the light and the disorder as captured by $\Sigma_{\text {IND }}^{R}$. This 
coherence was shown to be essential for a proper description of the propagators. It is equally important in the Eq. (72) for $G^{<}$. We compare for clarity the coherent parts $\operatorname{Im} G_{\mathrm{COH}}^{<}$with and without including $\Sigma_{\text {IND }}^{R}$ in Fig. 13. While the two surfaces are superficially similar, they differ markedly at long times. It should be noted that while $\Sigma_{\text {IND }}^{R}$ itself is a transient quantity, its coherent effects persist. This is well illustrated in Fig. 14 demonstrating the course of the full $\operatorname{Im} G^{<}$along the time diagonal. When (72) is solved without $\Sigma_{\text {IND }}^{R}$, the asymptotic value of the state occupancy differs from the full solution. This has important implications for the particle number conservation, as will be discussed in detail in the next section.

\section{PHYSICAL PROPERTIES OF THE SYSTEM}

In general, observable physical properties are given by the equal-time limit of the particle correlation function [1]. In the disorder case, this correspondence is not so simple in case the observable itself is represented by a random operator. For our model, however, the most important observables can be reduced to traces involving non-random operators.

\section{A. One-electron distribution and observables}

For each configuration, the one-electron density matrix at a time $t$ is given by $\varrho(t)=$ $\mathcal{S}\left(t, t_{0}\right) \varrho\left(t_{0}\right) \mathcal{S}\left(t_{0}, t\right)$. Thus, we have

$$
\begin{aligned}
& \varrho(t)=-\mathrm{i} \hbar \mathcal{G}^{<}(t, t) \\
& \rho(t) \equiv\langle\varrho(t)\rangle=-\mathrm{i} \hbar G^{<}(t, t)
\end{aligned}
$$

Consider now a one-electron observable represented by an operator $\mathcal{X}$, which must be taken as configuration dependent in general. Its mean value susceptible to measurement is given by a double average, configuration and quantum statistical. It must in general be written as

$$
\langle\langle\mathcal{X}\rangle\rangle=\operatorname{Tr}\langle\mathcal{X} \varrho\rangle
$$

Thus, the configuration average concerns the product $\mathcal{X} \varrho$ and canot be reduced to the knowledge of the configuration averaged $\rho$. The problem is similar to that of the random initial condition, and a generalized procedure for (86) depending on the structure of $\mathcal{X}$ is required. This problem will not be treated presently, as we will limit ourselves to the non-random observables.

\section{Averaged one-electron density matrix}

The configuration dependent equation of motion for $\varrho$ can be averaged with the result 


$$
\begin{aligned}
& \frac{\partial}{\partial t} \varrho(t)-\frac{1}{\mathrm{i} \hbar}\left[H_{\mathrm{MF}}(t), \varrho(t)\right]=\frac{1}{\mathrm{i} \hbar}[\mathcal{D}(t), \varrho(t)] \\
& \frac{\partial}{\partial t}\langle\varrho(t)\rangle-\frac{1}{\mathrm{i} \hbar} \underbrace{\left[H_{\mathrm{MF}}(t),\langle\varrho(t)\rangle\right]}_{\left.\mathrm{i} \hbar \frac{\partial}{\partial t}\langle\varrho(t)\rangle\right|_{\mathrm{DRIFT}}}=\frac{1}{\mathrm{i} \hbar}\{\underbrace{\Sigma^{R} G^{<}-G^{<} \Sigma^{A}}_{\left.\mathrm{i} \hbar \frac{\partial}{\partial t}\langle\varrho(t)\rangle\right|_{\mathrm{FORW}}}+\underbrace{\Sigma^{<} G^{A}-G^{R} \Sigma^{<}}_{\left.\mathrm{i} \hbar \frac{\partial}{\partial t}\langle\varrho(t)\rangle\right|_{\mathrm{BACK}}}\}_{t=t^{\prime}}
\end{aligned}
$$

The r.h.s. of the Eq. (88) represents the collision rate $\left.\frac{\partial}{\partial t}\langle\varrho(t)\rangle\right|_{\text {CoLL }}$ and the two terms correspond to the forward scattering and to the back scattering of the electrons. Altogether, a precursor kinetic equation is obtained, from which a true closed kinetic equation for $\rho$ may be derived [1]. This equation is not independent of the the equation of motion for $G^{<}$(and its conjugate), but it differs in the direction of integration in the $t, t^{\prime}$ plane: along the $t$ axis for (64), but along the time diagonal $t=t^{\prime}$ for Eq. (88). In a consistent theory, the Green functions and the self-energies computed directly should turn (88) into a tautology. At this point, we may only state that our numerically obtained Green functions obey the precursor equation (88), postponing all details to a publication about testing the ansatzes.

From (87), we see explicitly that in the exact theory the particle number is conserved, that is $\operatorname{Tr}\langle\varrho\rangle(t)$ is time independent. A proof will now be given that the SCBA does the same, as expected from a conserving approximation, $c f$. Subsection IVA3. By the Eq. (88), the conserving property is equivalent with the general criterion

$$
\operatorname{Tr}\left\{\Sigma^{R} G^{<}-G^{<} \Sigma^{A}+\Sigma^{<} G^{A}-G^{R} \Sigma^{<}\right\}_{t=t^{\prime}}=0
$$

An explicit derivation of the conserving property both for the SCBA and for the CPA was previously obtained [ $\mathbb{\theta}$ ] in the special case of a rectangular light pulse. Here, we verify that SCBA satisfies the Eq. (89) for an arbitrary pulse. The SCBA self-energy matrix is given by (60). With the propagators written as $G^{R}=\left(G^{>}-G^{<}\right) \vartheta\left(t-t^{\prime}\right)$ and $G^{A}=\left(G^{<}-G^{>}\right) \vartheta\left(t^{\prime}-t\right)$, the expression (89) becomes

$$
\begin{array}{r}
\left\langle\int _ { t _ { 0 } } ^ { t } \mathrm { d } \overline { t } \operatorname { T r } \left\{\mathcal { D } ( t ) \left(\left(G^{>}-G^{<}\right) \mathcal{D}(\bar{t}) G^{<}-G^{<} \mathcal{D}(\bar{t})\left(G^{<}-G^{>}\right)\right.\right.\right. \\
\left.\left.\left.+G^{<} \mathcal{D}(\bar{t})\left(G^{<}-G^{>}\right)-\left(G^{>}-G^{<}\right) \mathcal{D}(\bar{t}) G^{<}\right)\right\}\right\rangle
\end{array}
$$

The order of the linear operations $\langle\ldots\rangle, \int$ and Tr has been interchanged and the cyclic property of trace employed. The Green functions to the left of $\mathcal{D}(\bar{t})$ have arguments $(t, \bar{t})$, those to the right have $(\bar{t}, t)$. Clearly, the first term cancels with the fourth term, and the second with the third. The result is zero, as required.

\section{Observables and average values}

It will be convenient to compute the averages (86) in the Schrödinger picture; this amounts to the inverse transformation of the density matrix, 


$$
\begin{aligned}
\varrho_{\mathbf{S}} & =O^{\dagger} \varrho O \\
& =\varrho_{c c}+\varrho_{c v} \mathrm{e}^{-\mathrm{i} \Omega t}+\varrho_{v c} \mathrm{e}^{\mathrm{i} \Omega t}+\varrho_{v v}
\end{aligned}
$$

In the second line, the expression (7) for $O$ is used.

The total particle number is a special case of the average value of an observable $X=1_{\mathrm{op}}$, which is non-random, so that the averaged $\rho=\langle\varrho\rangle$ was sufficient to use in the preceding subsection. There is a number of other important observables which are non-random in our model. In particular, the electric polarization vector equals to

$$
\mathbf{P}=\Upsilon_{0}^{-1} N^{-1} \operatorname{Tr}\left(e \mathbf{r} \cdot\left\langle\varrho_{\mathbf{S}}\right\rangle\right)
$$

because the position vector is represented by the non-random off-diagonal operators $\mathbf{r} \rightarrow$ $\mathbf{r}_{c v}+\mathbf{r}_{v c}$. $\Upsilon_{0}$ denotes the volume of the primitive cell. The electric current density $\mathcal{J}=\Upsilon_{0}^{-1} N^{-1} e \dot{\mathbf{r}}=\Upsilon_{0}^{-1} N^{-1}(\mathrm{i} \hbar)^{-1}[e \mathbf{r}, \mathcal{H}]$, although a random operator, is also reduced to computing $\left\langle\rho_{\mathbf{S}}\right\rangle$, as

$$
\begin{aligned}
\langle\langle\mathcal{J}\rangle\rangle & =\Upsilon_{0}^{-1} N^{-1}\left\langle\operatorname{Tr}\left((\mathrm{i} \hbar)^{-1}[e \mathbf{r}, \mathcal{H}] \varrho_{\mathbf{S}}\right)\right\rangle \\
& =\Upsilon_{0}^{-1} N^{-1} \operatorname{Tr}(e \underbrace{\left\langle(i \hbar)^{-1}\left[\mathcal{H}, \varrho_{\mathbf{S}}\right]\right\rangle}_{\rho_{\mathbf{S}}}) \\
\mathbf{J} & =\frac{\partial}{\partial t} \mathbf{P}
\end{aligned}
$$

The energy transfer between the external disturbance and the system can similarly be simplified in our model in which the optical field has no random component, that is the local field corrections are negligible. In that case, the power absorbed per second in a unit volume is

$$
\begin{aligned}
w & =\mathbf{E} \cdot \mathbf{J} \\
& =\Upsilon_{0}^{-1} N^{-1} \operatorname{Tr}(\overbrace{\mathbf{E} \cdot e \mathbf{r}}^{-U(t)}\left\langle(\mathrm{i} \hbar)^{-1}\left[\mathcal{H}, \varrho_{\mathbf{S}}\right]\right\rangle) \\
& =\Upsilon_{0}^{-1} N^{-1} \operatorname{Tr}\left(\left\langle(\mathrm{i} \hbar)^{-1}[\underbrace{\mathcal{H}-U(t)}_{\equiv \mathcal{H}_{\text {DARK }}}, \mathcal{H}] \varrho_{\mathbf{s}}\right\rangle\right)
\end{aligned}
$$

We introduce the Hamiltonian $\mathcal{H}_{\mathrm{DARK}}$ of electrons in the dark sample. The final form of the absorbed energy rate is then

$$
w=\Upsilon_{0}^{-1} N^{-1} \frac{\partial}{\partial t}\left\langle\left\langle\mathcal{H}_{\mathrm{DARK}}\right\rangle\right\rangle
$$

The work done on the sample by the light turns into the change of the internal energy of the electrons, so that no true dissipation occurs, whether in the exact theory, or in the SCBA. This is to be expected in a theory without sources of dissipation. Naturally, this sets an upper limit on the time interval in which such purely elastic theory may be valid. 


\section{B. Computed one-electron properties}

The computed double time Green functions were presented in Sec. VQ. Here we continue with the results of numerical work considering the time-diagonal $-\mathrm{i} \hbar G^{<}(t, t)$, that is the oneelectron density matrix $\rho$.

\section{Time evolution of $\boldsymbol{\rho}(\boldsymbol{t})$}

First, we present in Fig. 15 the full density matrix (85) decomposed into its band- and $k$ - vector dependent components. Because in our parabolic band regime the distribution is isotropic in the $k$ space, we use the bare electron energy instead of $k$. In the figure, $\Delta=\epsilon_{c}(\mathrm{k})-\epsilon_{c}\left(\mathrm{k}^{\times}\right)$, where $\mathrm{k}^{\times}$corresponds to the weak field one photon resonance (see Fig. 2 and Eq. (25)). $\Delta$ is related to the usual detuning by a factor $m_{v} /\left(m_{c}+m_{v}\right)$. For the case presented, the spherical layer in the Brillouin zone corresponding to Fig. 15 is given by the inequalities $0.084 \leq|\mathrm{k}| \leq 0.103$ a.u., that is $0.134 \leq|\mathrm{k}| / k_{X} \leq 0.170$. Here, $k_{X}=2 \pi / a_{0}=0.605$ a.u.

The diagonal panels show the excitation into the conduction band and the corresponding depletion of the valence band. The band diagonal elements are real and equal to the occupation numbers $n_{c, v}(\mathrm{k})$. The overall effect corresponds to the expectation. A coherent transient excites a broad region in the BZ as an energy uncertainty effect. It is followed by the persistent excited population in a narrow strip around the resonance, which is the strongest for small detunings.

We use the off-diagonal panels to plot $\operatorname{Re} \rho_{c v}$ and $\operatorname{Im} \rho_{c v}$ which together give both offdiagonal elements $\rho_{c v}=\rho_{v c}^{*}$. This is the polarization part of the density matrix. It shows a complex oscillatory behavior with an approximate mirror symmetry around the zero detuning. The polarization is attenuated with the characteristic time $\hbar / \operatorname{Im} \Sigma_{\text {DARK }}^{R}(\mathrm{k})$, that is twice slower than the diagonal transient. This can be understood from (54) and (85) by a reasoning similar to that in Sec. $\mathrm{VC} 2$ : the relevant quantities to be compared are $G_{c v}^{R}\left(t, t_{0}\right) \varrho\left(t_{0}\right) G_{v c}^{A}\left(t_{0}, t\right)$ and, say, $G_{c v}^{R}\left(t, t_{0}\right) \varrho\left(t_{0}\right) G_{v v}^{A}\left(t_{0}, t\right)$. Both $G$ factors decay in the former expression, only one in the latter one. We note that we intentionally do not introduce a semi-empirical dephasing time leaving the loss of coherence entirely to the effects of disorder.

\section{Time evolution of observable properties}

Figure 16 presents, in a matrix arrangement, the total effect of the pulse integrated over the BZ,

$$
\rho_{a b}^{\mathrm{TOT}}(t)=N^{-1} \sum_{k} \rho_{a b}(\mathrm{k}, t), \quad a, b=c, v
$$

Full lines in all figures give the result of a direct GF computation. The diagonal panels show the total band populations per cell. An almost precise conservation law $\rho_{c c}^{\text {TOT }}+\rho_{v v}^{\text {TOT }}=1$ is a satisfactory result, because the two contributions are computed independently on the basis of constituents which do not show much resemblance individually. The coherent parts do not compensate (thin line), and the deficit is compensated for by the backscattered flow. 
Further, we return for the last time to the importance of the induced part of the particle self-energy, $\Sigma_{\mathrm{IND}}^{R}$. By a dotted line, we plot the result, if this self-energy component is neglected and thus the dark quasiparticle approximation is used. Very clearly, the particle number conservation is strongly violated, and the error occurs in the valence band. This is in good correspondence with the tests of the semi-group property in Sec. VB3, where the most pronounced deviations also occurred for the valence band Green function. This is most likely due to the fact that our valence band is not affected by the disorder directly, and its behavior is dictated by the indirect influence of the conduction band in a rather sensitive manner.

The off-diagonal component $\rho_{v c}^{\text {TOT }}$ is shown in the $v c$ corner of Fig. 16 by components, in the $c v$ corner in the semi-logarithmic form: $\rho_{v c}^{\text {Tот }}=R e^{\mathrm{i} \varphi}$. All quantities shown correspond to the Galitskii envelope. They are related to the induced polarization per cell by

$$
\begin{aligned}
\Upsilon_{0} \mathbf{P} & =e \mathrm{x}_{c v}\left\{\rho_{v c}^{\mathrm{TOT}} e^{-\mathrm{i} \Omega t}+\text { c. c. }\right\} \\
& =e \mathrm{x}_{c v} R(t) \cos (\Omega t+\varphi(t))
\end{aligned}
$$

Total polarization oscillates rapidly within the envelope $\pm R$. We compare its amplitude with the pulse envelope scaled to the rising part of polarization. The pulse and the response initially appear to be proportional having constant their ratio and phase difference. Around the pulse peak, the response is sub-linear, while the phase changes sign and rises close to $\frac{1}{2} \pi$. Polarization vanishes right after the pulse: practically all of the polarization is associated with the coherent part of the excitation. The rapid attenuation of the polarization is no doubt caused by a cancellation due to dephasing of the rapid oscillations of $\rho_{v c}(\mathrm{k}, t)$ seen in the previous figure.

Using Eqs. (95), (94) and (100), we get for the power absorbed per primitive cell and averaged over the $\Omega$ cycle

$$
\begin{aligned}
\Upsilon_{0} w & =2 Q \Phi(t)\left\{\operatorname{Re}\left(\frac{\partial}{\partial t} \rho_{v c}\right)-\Omega \operatorname{Im} \rho_{v c}\right\} \\
& =2 Q \Phi(t)\left\{\frac{\partial}{\partial t}(R \cos \varphi)-\Omega R \sin \varphi\right\}
\end{aligned}
$$

The $\Omega$ oscillations are rapid compared to variation of the Galitskii amplitudes (RWA condition) so that the second term in the braces is dominant. The phase angle between the current and the field is then basically $\varphi+\frac{1}{2} \pi$. It evolves from a small positive value $\approx 0.095 \pi$ to a saturation value close to $\pi$ : the energy is absorbed at first, then partly returned to the field, as shown in Fig 17. The integral $W=\int^{t} \mathrm{~d} t^{\prime} w\left(t^{\prime}\right)$ multiplied by $\Upsilon_{0}$ is the energy transferred between the pulse and one primitive cell up to the time $t$. It is also shown in the figure. The ratio $\Upsilon_{0} W(t) / \rho_{c c}^{\text {TOT }}(t)$ for times after the pulse gives the energy absorbed per a photoexcited electron; it corresponds closely to the basic frequency of the pulse, $\Omega=1.75 \mathrm{eV}$.

Considering the real magnitude of the photoexcitation, we have two electrons per primitive cell in the fully occupied valence band, that is the bulk electron density is $8 / a_{0}^{3}=3.3 \times 10^{27} \mathrm{~m}^{-3}$. With the fractional excitation $\sim 10^{-4}$, this yields $10^{23} \div 10^{24} \mathrm{~m}^{-3}$ of the electrons excited to the conduction band. Such densities are low enough to justify that we neglect the $e-e$ collisions during the time interval considered, $c f$. [2]. The induced polarization (93) can be estimated by $\mathrm{P}=8 / a_{0}^{3} \rho_{c v}^{\mathrm{TOT}} \cdot e a_{0}$ as compared with the vacuum electric induction $\mathrm{D}_{\mathrm{VAC}}=\varepsilon_{0} \mathrm{E}_{\mathrm{M}}$. Both quantities are comparable and have the $\sim 10^{-4} \mathrm{Cm}^{-2}$ order of magnitude. 


\section{CONCLUSION}

In this paper, we have addressed the problem of the response of electrons in semiconductors to a short light pulse. Of the three principal scattering mechanisms, we concentrated on the impurity scattering, and to make it pronounced, the case of concentrated semiconductor alloys was studied. For extremely short times, the disorder scattering in alloys may typically be dominant. The scattering regime is easily adjusted by varying the alloy composition.

The physical picture obtained has the following features:

$\diamond$ The alloy scattering in chemically saturated alloys is short range affecting thus large parts of the Brillouin zone.

$\diamond \mathrm{It}$ is elastic, which makes some coherence effects pronounced.

$\diamond$ Finally, it also acts in the dark, so that the dark polaron effect, i.e. use of dressed terminal states is essential. Typically, these dark states have a well-defined quasi-particle nature.

$\diamond$ The illuminated state cannot be reduced to a redistribution of electrons among the dark states, and the induced part of the electron self-energy describes a coherent time non-local coupling between the alloy scattering and the excitation process.

$\diamond$ As shown by a cumulant analysis, for very short times the dark component describing fast quasi-particle formation and the delayed induced component contribute to the self-energy additively, so that the formation process is insensitive to the illumination.

$\diamond$ The coherence between the light and scattering significantly affects the photoexcited distribution; in particular, it conditions the particle number and energy conservation.

All these conclusions are obtained analytically and confirmed numerically.

The paper discusses also technical questions. The NGF describing the process are obtained by a direct solution of equations equivalent to the Kadanoff-Baym equations. The asymmetric LW choice of computing first the propagators and using these as an input in the equation for the particle correlation function is advantageous in the present case of an elastic scattering. The isotropic scattering would require $k$-integration over the whole BZ. This is overcome by separating out the dark self-energy and integrating explicitly only the induced part, stemming from a narrow slice around the resonance in the $k$-space. To suppress rapid oscillations of the integrand, we work in the Galitskii representation, in which the pulse is represented by its envelope, while the high basic frequency enters only as a relative shift of the bands.

For the future work, this paper suggests several directions as an immediate sequel:

(i) It will be important to extend the NGF approach to the Coherent potential approximation, which is a self-consistent intermediate theory suitable for all realistic alloy scattering strengths.

(ii) Then random ("correlated") initial conditions will play a non-trivial role, and should be incorporated.

(iii) These analytical approximations for the NGF may be, in the alloy case, compared with a direct simulation for explicitly generated random alloy configurations and a numerically performed configuration average.

Independently of these extensions and modifications, the results of the present work can readily be used for an analysis of various methods to develop quantum kinetic equations based on an ansatz generalizing the KBA, and for a comparison of the resulting approximate solutions with the NGF results. Such analysis should serve to find the validity limits of the 
individual ansatzes and the underlying physical explanation. A communication concerning these questions is also in preparation.

\section{ACKNOWLEDGMENT}

This work was supported by the Grant Agency of the Czech Republic under the project number 202/00/0643.

\section{APPENDIX A: $G^{<}$WITH INITIAL CORRELATIONS}

Like in the main text, the basic definition of $G^{<}$is the Eq. (52), but now with the initial condition (53) at the initial moment $t_{0}$ being random. We express the propagators as $\mathcal{G}^{R}=G^{R}+G^{R}\left(\mathcal{D}-\Sigma^{R}\right) \mathcal{G}^{R}, \mathcal{G}^{A}=G^{A}+\mathcal{G}^{A}\left(\mathcal{D}-\Sigma^{A}\right) G^{A}$. The expresssion $G^{<}=\mathrm{i} \hbar\left\langle\mathcal{G}^{R}{ }_{o} \rho_{o} \mathcal{G}^{A}\right\rangle$ becomes

$$
\begin{aligned}
G^{<} & =\mathrm{i} \hbar\left(G^{R}{ }_{\left.{ }_{o} \rho_{o}\right\rangle} G^{A}\right. \\
& +G^{R}\left\langle{ }_{o} \rho_{o} \mathcal{G}^{A}\left(\mathcal{D}-\Sigma^{A}\right)\right\rangle G^{A}+G^{R}\left\langle\left(\mathcal{D}-\Sigma^{R}\right) \mathcal{G}^{R}{ }_{o} \rho_{o}\right\rangle G^{A} \\
& \left.+G^{R}\left\langle\left(\mathcal{D}-\Sigma^{R}\right) \mathcal{G}^{R}{ }_{o} \rho_{o} \mathcal{G}^{A}\left(\mathcal{D}-\Sigma^{A}\right)\right\rangle G^{A}\right) \\
& \quad\left(t \geq t_{0}, t^{\prime} \geq t_{0}\right)
\end{aligned}
$$

The three lines of (A2) correspond to an averaged ("uncorrelated") initial condition, to the random field analogue of the correlated initial conditions, and to the genuine particle correlation, respectively. These terms have the same general structure $G^{R} \ldots G^{A}$, but they differ in the number of inner times equal to $t_{0}$, two, one, and none. We make this explicit using the "o" subscripts and define

$$
\begin{aligned}
{ }_{o} \Lambda^{<} & \equiv \mathrm{i} \hbar\left\langle{ }_{o} \rho_{o} \mathcal{G}^{A}\left(\mathcal{D}-\Sigma^{A}\right)\right\rangle \\
\Lambda_{o}^{<} & \equiv \mathrm{i} \hbar\left\langle\left(\mathcal{D}-\Sigma^{R}\right) \mathcal{G}^{R}{ }_{o} \rho_{o}\right\rangle \\
\Sigma^{<} & \equiv\left\langle\left(\mathcal{D}-\Sigma^{R}\right) \mathcal{G}^{<}\left(\mathcal{D}-\Sigma^{A}\right)\right\rangle
\end{aligned}
$$

With these definitions, the final form of $G^{<}$is

$$
G^{<}=\mathrm{i} \hbar G^{R}\left\langle\rho_{o}\right\rangle G^{A}+G^{R} \Lambda^{<} G^{A}+G^{R} \Lambda_{o}^{<} G^{A}+G^{R} \Sigma^{<} G^{A}
$$

This equation extends the Eq. (54) by incorporating a memory effect caused by the disorder induced initial correlations. In all other respects, comments made to (54) apply here equally well.

The diferential equation analogous to the Dyson equation (64) is obtained, if we differentiate (A6) from the left, and employ (61) for $G^{R}$ in the form

$$
\left(\mathrm{i} \hbar \partial_{t}-H_{\mathrm{MF}}\right) G^{R}=\Sigma^{R} G^{R}+I .
$$

For $t>t_{0}$, the $\delta$-singularity is effective for only two terms of (A6), and the resulting equation reads 


$$
\left(\mathrm{i} \hbar \partial_{t}-H_{\mathrm{MF}}\right) G^{<}=\Sigma^{R} G^{<}+\Sigma^{<} G^{A}+\Lambda_{o}^{<} G^{A}
$$

The two seemingly missing terms reappear in the initial condition:

$$
G^{<}\left(t_{0}, t^{\prime}\right)=\left[\left\langle{ }_{o} \rho_{o}\right\rangle G^{A}+{ }_{o} \Lambda^{<} G^{A}\right]_{t=t_{0}, t^{\prime}}
$$

It remains to develop an approximation scheme for the self-energy, both for the regular part $\Sigma^{<}$and for the singular part $\Lambda^{<}+\Lambda_{o}^{<}$. The two $\Lambda^{\prime}$ 's are equivalent:

$$
{ }_{o} \Lambda^{<}\left(t, t^{\prime}\right)=-\left\{\Lambda_{o}^{<}\left(t^{\prime}, t\right)\right\}^{\dagger}
$$

so that the whole $<$ component of the self-energy obeys the symmetry (43). Considering, say, $\Lambda_{o}^{<}$, we see from (A4) that it only involves retarded quantities, which do not depend

on the initial conditions for elastic scattering. Thus, $\Lambda_{o}^{<}$is a linear functional of the initial distribution $\rho_{0}$, and it does not involve any particle-hole correlation. In other words, $\Lambda$ 's can computed on the propagator level. The averaging procedure leading to (A4) depends on the initial distribution, however.

The Eq. (A5) for $\Sigma^{<}$does not explicitly depend on the initial condition. In the SCBA, it will have the form $\Sigma_{\mathrm{SCBA}}^{<}=\left\langle\mathcal{D} G^{<} \mathcal{D}\right\rangle$ identical with (66), but with $G^{<}$given by (A6) and (A8). Only in higher order approximations, the genuine correlations of the three-terminal type, like $\left\langle\mathcal{D} G^{R}\left({ }_{o} \rho_{o}-\left\langle{ }_{o} \rho_{o}\right\rangle\right) G^{A} \mathcal{D}\right\rangle$, start playing their role, and must be systematically accounted for.

\section{APPENDIX B: RANDOM OBSERVABLES}

Let us return to (86), but write it in the Schrödinger picture, $c f$. (92):

$$
\langle\langle\mathcal{X}\rangle\rangle=\operatorname{Tr}\left\langle\mathcal{X} \varrho_{\mathbf{S}}\right\rangle
$$

If the observable $\mathcal{X}$ is random itself, the double average has to be performed at the end, as discussed in the main text. The average can be given two different forms:

$$
\begin{aligned}
\langle\langle\mathcal{X}\rangle\rangle_{t} & =\hbar^{2} \operatorname{Tr}\left\langle\mathcal{X} \mathcal{G}^{R}\left(t, t_{0}\right)_{o} \rho_{o} \mathcal{G}^{A}\left(t_{0}, t\right)\right\rangle \\
& =-\mathrm{i} \hbar \operatorname{Tr}\left\langle\mathcal{X} \mathcal{G}^{<}\left(t, t^{\prime} \rightarrow t\right)\right\rangle
\end{aligned}
$$

The first form, Eq. (B1), makes explicit that the random initial condition and a random observable enter the averages in a symmetrical way, and any general technique for their evaluation has to be built up symmetrically. The second form of $\langle\langle\mathcal{X}\rangle\rangle$, seemingly trivial, replaces the density matrix by the particle correlation function, and this may lead to explicit averaging procedures in special cases.

As an important example, let us consider the dark Hamiltonian in our model and calculate $\left\langle\left\langle\mathcal{H}_{\text {DARK }}\right\rangle\right\rangle$, a quantity related to the Joule work by (97). This does not go with $\langle\rho\rangle$, but we may proceed as follows: 


$$
\begin{aligned}
\mathrm{i} \hbar \frac{\partial}{\partial t} \mathcal{G}^{<} & =\left(\mathcal{H}_{\mathrm{DARK}}+U(t)\right) \mathcal{G}^{<} \\
\left\langle\mathcal{H}_{\mathrm{DARK}} \mathcal{G}^{<}\right\rangle & =\mathrm{i} \hbar \frac{\partial}{\partial t} G^{<}-U(t) G^{<} \\
\left\langle\left\langle\mathcal{H}_{\mathrm{DARK}}\right\rangle\right\rangle_{t} & =\operatorname{Tr}\left\langle\mathcal{H}_{\mathrm{DARK}}(-\mathrm{i} \hbar) \mathcal{G}^{<}\left(t, t^{\prime} \rightarrow t\right)\right\rangle \\
& =\hbar^{2} \operatorname{Tr}\left(\frac{\partial}{\partial t} G^{<}\left(t, t^{\prime} \rightarrow t\right)\right)-\operatorname{Tr}(U(t) \rho)
\end{aligned}
$$

Use is made of the fact that $U$ is non-random, and its average reduces to the use of $\rho=\langle\varrho\rangle$. The essential point is, however, that the derivative on the r.h.s. is NOT a derivative of the density matrix. The trick is similar to the computation of correlation energy using the one-particle GF in usual many-body theory. The derivative can be found directly (it would be enough to store it during the solution of the Dyson eq. for $G^{<}$), or it could be expressed using this DE (see Eq. (64)).

Thus, we can do two things: either get $\left\langle\left\langle\mathcal{H}_{\mathrm{DARK}}\right\rangle_{t}\right.$ directly from (B3), or express it in terms of the integral of the "Joule heat", $W(t)=\int^{t} \mathrm{~d} t^{\prime} w\left(t^{\prime}\right)$, using (97). The two results should be the same. This provides another criterion for the conserving nature of any approximations involved in the calculation. 


\section{REFERENCES}

[1] H. Haug, and A.P. Jauho,Quantum Kinetics in Transport and Optics of Semiconductors,(Springer, Berlin Heidelberg 1997), Vol.123

[2] M. Bonitz,Quantum Kinetic Theory,(Teubner, Stuttgart Leipzich 1998)

[3] L.P. Kadanoff, and G. Baym,Quantum Statistical Mechanics,(Benjamin, New York, 1962)

[4] L.V. Keldysh, Zh.exper.teor.Fiz.,47, 1515 (1964); translation: Soviet Physics JETP 20,1018 (1965)

[5] P. Danielewicz, Ann. Phys. (N.Y.) 152, 305 (1984); P. Danielewicz, Ann. Phys. (N.Y.) 152,239 (1984)

[6] H.S. Köhler, N.H. Kwong, R. Binder, D. Semkat, and M. Bonitz: in Progress in NonEquilibrium Greeen's Function, ed.M. Bonitz (World Scientific, Singapore 2000), p.464

[7] Progress in Non-Equilibrium Greeen's Function, ed. M. Bonitz (World Scientific, Singapore 2000)

[8] A. Kalvová, and B. Velický, Z. Phys B 94,273 (1994)

[9] B. Velický and A. Kalvová, phys. stat. sol. (b) 188, 515 (1995)

[10] A. Kalvová, and B. Velický, Z. Phys B 103, 33 (1997)

[11] B. Velický and A. Kalvová, phys. stat. sol. (b) 206,341 (1998)

[12] B. Velický, and A. Pieczonková, Phys. Scri. T19,558,(1987)

[13] A. Gonis, Green Functions for Ordered and Disordered Systems,Studies in Math.Phys. Vol. 4 (North Holland, Amsterdam 1992)

[14] W. Kohn, and J. Luttinger, Phys.Rev.108,590 (1957); J. Luttinger, and W. Kohn, Phys.Rev.109,1892 (1958)

[15] P. Lipavský, V. Špička, and B. Velický, Phys. Rev.B 34, 6933 (1986)

[16] D. Langreth, and J.W. Wilkins, Phys. Rev. B 6, 3189 (1972)

[17] A. -B. Chen, and A. Sher, Phys.Rev. B 23, 5360 (1981)

[18] K.C. Hass, H. Ehrenreich, and B. Velick y, Phys.Rev. B 27, 1088 (1983)

[19] W.A. Harrison, Electronic Structure and the Properties of Solids, (W.H. Freeman, San Francisco 1980)

[20] P.Y. Yu, and M. Cardona,Fundamentals of Semiconductors,(Springer Verlag, Berlin Heidelberg 1999)

[21] V.M. Galitskii, S.P. Goreslavskii, and V.F. Elesin, Soviet.Phys. JETP 30, 117 (1970)

[22] P. Gartner, L. Bányai, and H. Haug, Phys.Rev. B 60, 14234 (1999)

[23] R. Brout and P. Carruthers, Lectures on the many-electron problem, (Interscience Publishers, New York-London-Sydney 1963)

[24] G.D. Mahan, Many-Particle Physics,(Plenum Press, New-York and London 1990)

[25] B. Velický,S. Kirkpatrick, and H. Ehrenreich, Phys. Rev. 175,747 (1968)

[26] L. Banyai, H. Haug, and P. Gartner, Eur. Phys. J. B 1, 209 (1998)

[27] Ping Sheng,Scattering and localization of classical waves in random media,(Academic Press, New York 1995)

[28] D. Semkat, D. Kremp, and M. Bonitz, Phys. Rev. E59,1557 (1999)

[29] $0.88137=(-\log (\sqrt{ } 2-1)) ; 1.21169=(\log (\sqrt[4]{2}+\sqrt{\sqrt{ } 2-1})-\log (\sqrt[4]{2}-\sqrt{\sqrt{ } 2-1}))$ 


\section{TABLES}

TABLE I. Concentration dependence of the alloy electron structure: weak field one photon resonant energy, level broadening and particle lifetime

\begin{tabular}{lccr}
\hline \hline$c$ & $\hbar \Omega^{\times} / \mathrm{eV}$ & $\left|\operatorname{Im} z_{k}\right| / \mathrm{eV}$ & $\tau / \mathrm{fs}$ \\
\hline 0.00 & 1.80 & 0.0000 & $\infty$ \\
0.05 & 1.75 & 0.0020 & 165 \\
0.15 & 1.63 & 0.0059 & 53 \\
0.50 & 1.34 & 0.0105 & 31 \\
& & & \\
\hline \hline
\end{tabular}

TABLE II. Lowest moments and cumulants: exact and in the SCBA

\begin{tabular}{ccccc}
\hline \hline $\boldsymbol{p}$ & $\boldsymbol{M}_{\boldsymbol{p}}$ SCBA & $\boldsymbol{M}_{\boldsymbol{p}}$ EXACT & $\boldsymbol{C}_{\boldsymbol{p}}$ SCBA & $\boldsymbol{C}_{\boldsymbol{p}}$ EXACT \\
\hline 0 & 1 & 1 & 1 & 1 \\
1 & 0 & 0 & 0 & 0 \\
2 & $\gamma$ & $\gamma$ & $\gamma$ & $\gamma$ \\
3 & $\gamma \mu_{1}$ & $\gamma \mu_{1}+\gamma\left(c_{B}-c_{A}\right) \delta$ & $\gamma \mu_{1}$ & $\gamma \mu_{1}+\gamma\left(c_{B}-c_{A}\right) \delta$ \\
4 & $\gamma \mu_{2}+\mathbf{2} \gamma^{2}$ & $\gamma \mu_{2}+2 \gamma\left(c_{B}-c_{A}\right) \delta \mu_{1}+$ & $\gamma \mu_{2}-\mathbf{1} \gamma^{2}$ & $\gamma \mu_{2}+2 \gamma\left(c_{B}-c_{A}\right) \delta \mu_{1}+$ \\
& & $+\gamma\left(\delta^{2}-\mathbf{3} \gamma\right)$ & & $+\gamma\left(\delta^{2}-\mathbf{6} \gamma\right)$
\end{tabular}

TABLE III. Characteristic parameters of the pulse

\begin{tabular}{lcccr}
\hline \hline$t_{\mathrm{P}}[\mathrm{ps}]$ & $Q[\mathrm{eV}]$ & $\mathrm{E}_{\mathrm{M}}\left[\mathrm{Vm}^{-1}\right]$ & $t_{\mathrm{FWHM}}[\mathrm{ps}]$ & $\varphi_{\mathrm{R}}$ \\
\hline 0.1 & 0.01 & $1.89 \times 10^{7}$ & 0.121 & $1.93 \pi$ \\
& & & & \\
\hline \hline
\end{tabular}




\section{FIGURES}

FIG. 1. Local Green function $F_{o}(E+\mathrm{i} 0)$ of the pure A crystal for energies between the band edges $1.5 \mathrm{eV}$ and $13.5 \mathrm{eV}$ on the real axis.

FIG. 2. Renormalized dispersion law $z_{k}$ as a function of bare energy $\epsilon_{k}$ for four values of concentration $c=0 . ; 0.05 ; 0.17 ; 0.5$. Dotted lines: mean field rigid shift of the whole band. Thick line: full dispersion law with polaron shift included. Energy dependent band broadening shown by lining each of the quasi-particle energy by thin line at a distance $\pm \operatorname{Im} z_{k}$. Dot-dashed lines: shifted valence band $\epsilon_{v}(\mathrm{k})+\hbar \Omega^{\times}(c)$.

FIG. 3. Renormalization constant as a function of bare energy for the same values of concentration as in Fig.2. Upper part (thick lines): modulus $\left|Z_{k}\right|-1$. Lower part (thin lines): phase $\arg \left(Z_{k}\right)$.

FIG. 4. Evolution of the "action" $\varsigma(t)$ for dark propagator on femtosecond time scale. Fig. 4a: real part, Fig. 4b: imaginary part. Full line: full SCBA solution. Dashed line: pole approximation (30) in time domain. Detail for the shortest times $\leq 0.3 \mathrm{fs}$ (small windows) expanded in the insert in the left lower corner and compared with the lowest (full dots) and next lowest (open dots) order cumulant expansions. The insert in the right upper corner: effective self-energy $\sigma_{k}$, Eq. (40) (full line) tends rapidly to the constant quasi-particle (pole) energy (dashed line).

FIG. 5. Schematic flow chart for calculation of $\Sigma_{I}^{R}\left(t, t^{\prime}\right)$. Input: dark QP for $p=0$. Iteration counter: $p$. Output: $\Sigma_{I}^{R}\left(t, t^{\prime}\right)$ and $G^{R}\left(\mathrm{k} ; t, t^{\prime}\right)$ (not indicated).

FIG. 6. Schematic flow-chart for calculation of $\Sigma^{<}\left(t, t^{\prime}\right)$. Input: propagator components $\Sigma_{\mathrm{D}}^{R}$, $\Sigma_{\text {IND }}^{R}, G^{A}$. Output: $\Sigma^{<}\left(t, t^{\prime}\right)$ (and $G^{<}\left(\mathrm{k} ; t, t^{\prime}\right)$, if required - not shown). Main differences with respect to $\Sigma_{\text {IND }}^{R}\left(t, t^{\prime}\right)$ (see Fig.5): $\diamond$ non-iterative process (see text); $\diamond$ the initial time $t^{\prime} \ldots$ outermost loop, the current time $t \ldots$ innermost loop. This "normal" order of loops was reverted for $\Sigma^{R}$.

FIG. 7. Imaginary part of the induced self-energy (73) as a function of both time variables. Insert: the same plot rotated to the view in the direction of the arrow (along the $t+t^{\prime}$ axis). Vertical scale unit: $\mathrm{eV} / \mathrm{ps}$.

FIG. 8. Band diagonal elements $\left|\mathrm{i} \hbar G_{b b}^{R}\right|^{2}$ for resonant wave vector $\mathrm{k}^{\times}$as a function of $t$ and $t^{\prime}$. Upper panel: $b=c$. Lower panel: $b=v$. In each panel, two surfaces are marked by dots, the full SCBA $G^{R}$, and its approximation with $\Sigma_{\mathrm{I}}^{R}$ neglected. As an aid for eye, the exact surface has several lines $t^{\prime}=$ const marked by heavier points. The dot patterns permit to view the surfaces either in the $t, t^{\prime}$ coordinates, or in the Wigner coordinates $t \pm t^{\prime}$. 
FIG. 9. Test of the semigroup multiplicative property (83). Heavy line: i $\hbar G^{R}(t,-0.2)$. Thin lines: $\mathrm{i} \hbar G^{R}\left(t, t^{\prime \prime}\right) \mathrm{i} \hbar G^{R}\left(t^{\prime \prime},-0.2\right)$ for $t>t^{\prime \prime}$. Quantity plotted: $\left|i \hbar G_{c c}\right|^{2}+\left|i \hbar G_{v c}\right|^{2}$ (upper panels) and $\left|i \hbar G_{v v}\right|^{2}+\left|i \hbar G_{c v}\right|^{2}$ (lower panels). The pulse envelope (可) and the factorization times $t$ " (squares) shown at the top. The first, third, and fifth $t$ " were singled out also in Fig. 8. Left hand column: full SCBA, $\Sigma_{\text {ind }}\left(t, t^{\prime}\right)$ included. Right hand column: WW approximation, $\Sigma_{\text {ind }}\left(t, t^{\prime}\right)$ neglected. Here, the factorization is valid and the thin lines merge with the basic heavy plot.

FIG. 10. Self-energy $\Sigma^{<}\left(t, t^{\prime}\right)$ as a self-consistent result of eqs. (72) and (77). Upper panel: real part, lower panel: imaginary part. Inserts: the same plot viewed in the direction of the arrow (along the $t+t^{\prime}$ axis). Units of $\Sigma: \mathrm{eV} / \mathrm{ps}$.

FIG. 11. The particle correlation function $G_{c c}^{<}\left(\mathrm{k} ; t, t^{\prime}\right)$ for resonant detuning $\mathrm{k}=\mathrm{k}^{\times}$. Upper panel: real part, lower panel: imaginary part. Inserts: view along the arrow - as in Fig.10. Units: $\hbar G$ is dimensionless

FIG. 12. $\operatorname{Im} G_{c c}^{<}\left(\mathrm{k}^{\times} ; t, t^{\prime}\right)$ of the previous figure split into its coherent (upper panel) and incoherent (lower panel) parts according to (54).

FIG. 13. The coherent part $\operatorname{Im} G_{c c, \mathrm{COH}}^{<}\left(\mathrm{k}^{\times} ; t, t^{\prime}\right)$ of Fig. 12 (upper surface, coarse grid) contrasted with the calculation, where $\Sigma_{\text {IND }}^{R}\left(t, t^{\prime}\right)$ was neglected.(lower surface, fine grid).

FIG. 14. Time diagonal $\operatorname{Im} G_{c c}^{<}\left(\mathrm{k}^{\times} ; t, t^{\prime}=t\right)$. Thick line: data of Fig.11. Thin line: the same quantity, but $\Sigma_{\mathrm{IND}}^{R}$ neglected in computation.

FIG. 15. One-electron density matrix decomposed into its band and $k$-vector dependent components. Detuning $\Delta$ equals to the excess energy $\epsilon_{c}(\mathrm{k})-\epsilon_{c}\left(\mathrm{k}^{\times}\right)$measured with respect to the one photon resonance. Normalization to one primitive cell, $\rho$ is dimensionless. The $\Delta=0$ profile identical with the thick line of Fig. 14

FIG. 16. Total photoexcitation $\rho_{a b}^{\text {TOT }}$, Eq. (98). Diagonal panels: total band population per cell (thick line ... direct GF computation; thin line ... coherent part only; dashed line ... computation neglecting $\Sigma_{\text {IND }}^{R}$ ). Conservation law $\rho_{c c}^{\text {TOT }}+\rho_{v v}^{\text {TOT }}=1$ : very good for direct GF computation, poor in both other cases. Off-diagonal panels: The $v c$ corner: the off-diagonal component $\rho_{v c}^{\text {TOT }}$. The $c v$ corner: the same quantity in semi-logarithmic form $\rho_{v c}^{\mathrm{TOT}}=R \mathrm{e}^{\mathrm{i} \varphi}$. Thick line $\ldots R$. Thin line $\ldots \varphi$. Dotted line ... pulse envelope $\Phi$ scaled to coincide with $R$ at early times. Note the good fit. All quantities shown are dimensionless.

FIG. 17. Power absorbed per primitive cell and averaged over the $\Omega$ cycle ... thick line; integral energy transfer between the pulse and one primitive cell ... thin line Units: $\mathrm{eV} / \mathrm{ps}$ for $\Upsilon_{0} w$, eV for $\Upsilon_{0} W$. 
This figure "Fig1.gif" is available in "gif" format from: http://arxiv.org/ps/cond-mat/0202113v1 
This figure "Fig2.gif" is available in "gif" format from: http://arxiv.org/ps/cond-mat/0202113v1 
This figure "Fig3.gif" is available in "gif" format from: http://arxiv.org/ps/cond-mat/0202113v1 
This figure "Fig4a.gif" is available in "gif" format from: http://arxiv.org/ps/cond-mat/0202113v1 
This figure "Fig4b.gif" is available in "gif" format from: http://arxiv.org/ps/cond-mat/0202113v1 
This figure "Fig5.gif" is available in "gif" format from: http://arxiv.org/ps/cond-mat/0202113v1 
This figure "Fig6.gif" is available in "gif" format from: http://arxiv.org/ps/cond-mat/0202113v1 
This figure "Fig7.gif" is available in "gif" format from: http://arxiv.org/ps/cond-mat/0202113v1 
This figure "Fig8.gif" is available in "gif" format from: http://arxiv.org/ps/cond-mat/0202113v1 
This figure "Fig9.gif" is available in "gif" format from: http://arxiv.org/ps/cond-mat/0202113v1 
This figure "Fig10.gif" is available in "gif" format from: http://arxiv.org/ps/cond-mat/0202113v1 
This figure "Fig11.gif" is available in "gif" format from: http://arxiv.org/ps/cond-mat/0202113v1 
This figure "Fig12.gif" is available in "gif" format from: http://arxiv.org/ps/cond-mat/0202113v1 
This figure "Fig13.gif" is available in "gif" format from: http://arxiv.org/ps/cond-mat/0202113v1 
This figure "Fig14.gif" is available in "gif" format from: http://arxiv.org/ps/cond-mat/0202113v1 
This figure "Fig15.gif" is available in "gif" format from: http://arxiv.org/ps/cond-mat/0202113v1 
This figure "Fig16.gif" is available in "gif" format from: http://arxiv.org/ps/cond-mat/0202113v1 
This figure "Fig17.gif" is available in "gif" format from: http://arxiv.org/ps/cond-mat/0202113v1 